\title{
Hybrid fs/ps rotational CARS temperature and oxygen measurements in the product gases of canonical flat flames
}

\author{
Sean P. Kearney \\ Engineering Sciences Center \\ Sandia National Laboratories \\ Albuquerque, NM 87185 \\ Submitted to Combustion and Flame
}

Contact information:

Sean P. Kearney

Sandia National Laboratories

PO Box 5800, Mail Stop 0826

Albuquerque, NM 87185-0826

Voice: +1-505-844-6669

Mobile: +1-505-270-0729

Email: spkearn@sandia.gov

Keywords: Laser diagnostics; femtosecond CARS; flat flames; temperature; oxygen 


\section{Abstract}

A hybrid fs/ps pure-rotational coherent anti-Stokes Raman scattering (CARS) scheme is systematically evaluated over a wide range of flame conditions in the product gases of two canonical flat-flame burners. Near-transform-limited, broadband femtosecond pump and Stokes pulses impulsively prepare a rotational Raman coherence, which is later probed using a highenergy, frequency-narrow picosecond beam generated by the second-harmonic bandwidth compression scheme that has recently been demonstrated for rotational CARS generation in $\mathrm{H}_{2} /$ air flat flames. The measured spectra are free of collision effects and nonresonant background and can be obtained on a single-shot basis at $1 \mathrm{kHz}$. The technique is evaluated for temperature/oxygen measurements in near-adiabatic $\mathrm{H}_{2}$ /air flames stabilized on the Hencken burner for equivalence ratios of $\phi=0.20$ to 1.20 . Thermometry is demonstrated in hydrocarbon/air products for $\phi=0.75$ to 3.14 in premixed $\mathrm{C}_{2} \mathrm{H}_{4} /$ air flat flames on the McKenna burner. Reliable spectral fitting is demonstrated for both shot-averaged and single-laser-shot data using a simple phenomenological model. Measurement accuracy is benchmarked by comparison to adiabatic-equilibrium calculations for the $\mathrm{H}_{2}$ /air flames, and by comparison with nanosecond CARS measurements for the $\mathrm{C}_{2} \mathrm{H}_{4}$ /air flames. Quantitative accuracy comparable to nanosecond rotational CARS measurements is observed, while the observed precision in both the temperature and oxygen data is extraordinarily high, exceeding nanosecond CARS, and on par with the best published thermometric precision by femtosecond vibrational CARS in flames, and rotational femtosecond CARS at low temperature. Threshold levels of signal-to-noise ratio to achieve $1-2 \%$ precision in temperature and $\mathrm{O}_{2} / \mathrm{N}_{2}$ ratio are identified. The results show that purerotational fs/ps CARS is a robust and quantitative tool when applied across a wide range of flame conditions spanning lean $\mathrm{H}_{2}$ /air combustion to fuel-rich sooting hydrocarbon flames. 


\section{Introduction}

Coherent anti-Stokes Raman scattering (CARS) is a powerful diagnostic for temperature and species detection in combustion [1]. The technique is particularly well-renowned for highprecision thermometry, robust applicability in luminous sooting flames, and good performance in large-scale technical combustion systems where many other laser diagnostics struggle [2-7]. CARS instruments for combustion have most often been based on Nd:YAG and dye laser setups that provide nanosecond-duration laser pulses, but there has been a recent surge in research [827] investigating the utility of femtosecond laser sources for potentially dramatic leaps forward in combustion diagnostics. Commercial femtosecond amplifiers offer $\mathrm{kHz}$-scale repetition rates, allowing researchers to dramatically exceed the $\sim 10 \mathrm{~Hz}$ barrier posed by high-energy nanosecond laser systems, and approach the energy containing scales of some turbulent flames [23, 26]. Femtosecond CARS approaches permit complete time-gated elimination of nonresonant background contributions, which is particularly helpful in fuel-rich flames [28]. The need for detailed Raman linewidth information, which is often uncertain in turbulent flames, is reduced or eliminated because some femtosecond CARS schemes are insensitive to collisional effects at pressures perhaps as high as 30-50 bar [16, 29]. Time-delayed probing of femtosecond-prepared Raman coherences additionally permits in situ linewidth determination [3, 15, 21, 30-32], which may be particularly useful in high-pressure flames. Femtosecond sources provide nearly transform-limited pump and Stokes pulses, whose large bandwidth and well-defined spectral phase relationship allow for highly efficient [9] preparation of the Raman coherence with very low noise, which has resulted in single-shot temperature-measurement precision in flames as good as $1 \%$ [24] when vibrational CARS of $\mathrm{N}_{2}$ has been employed. 
Pure-rotational CARS provides distinct advantages for combustion monitoring and has been the subject of recent development $[14,15,17-22,27,33-37]$ for use with femtosecond pump/Stokes laser schemes. Rotational Raman spectra are a highly sensitive thermometer over a wide range of temperatures [38-40], and offer a potentially straightforward approach to simultaneous species detection because rotational transitions of many combustion-relevant molecules $\left(\mathrm{N}_{2}, \mathrm{O}_{2}, \mathrm{CO}, \mathrm{CO}_{2}\right)$ lie below $\sim 300 \mathrm{~cm}^{-1}$ and are accessible with a single broadband laser source [41, 42]. The fs/ps pure-rotational CARS process employed here is illustrated in the energy level diagram of Figure 1. A rotational Raman coherence is impulsively prepared by broadband femtosecond pump and Stokes pulses which arrive nearly simultaneously at time $t=$ 0. The evolving coherence is then probed by a frequency-narrow picosecond pulse that is introduced at delay $t=\tau$ to yield frequency-resolved resolved Raman spectra at the completion of this "hybrid" fs/ps scheme [43].

For rotational CARS thermometry, three complementary approaches have been demonstrated to provide a frequency-narrow picosecond probe that is phase-locked to the femtosecond pump/Stokes preparation. The method demonstrated by Kliewer and Bohlin [19, 20, 27, 33, 34] utilizes a high-energy $\mathrm{Nd}$ :YAG regenerative amplifier that is RF locked to a femtosecond Ti:sapphire laser to deliver $\sim 90$-ps duration probe beams with pulse energies of several 10s of mJ at $20-\mathrm{Hz}$ rates. These high-energy, narrow-linewidth probe beams result in very high CARS signal strength, so that flame spectra are routinely acquired along 1-D lines [20, 27, 34], and 2-D CARS imaging in flames has recently demonstrated [19, 33]. The relatively long duration of these probe pulses is comparable to coherence dephasing times, which renders these CARS spectra sensitive to collisions, and the data rate is currently limited to $20-\mathrm{Hz}$ with this approach. 
To retain the advantages of collision-free detection and $\mathrm{kHz}-\mathrm{level}$ data rates for rotational spectroscopy, two hybrid fs/ps CARS schemes have featured sub-10-ps probe pulses that are phase locked to the femtosecond pump/Stokes preparation. This has been most readily achieved by simply removing bandwidth from a portion of the femtosecond source, as demonstrated by Prince et al. [43], and successfully applied for vibrational fs/ps CARS thermometry in flames by Miller et al. [12]. Single-laser-shot rotational fs/ps gas-phase CARS thermometry based on direct bandwidth removal has been demonstrated by Miller et al. [14, 16], Stauffer et al. [22] and Kearney et al. [17]. Unfortunately, single-shot thermometry was limited to temperatures below $T$ $=800 \mathrm{~K}$ because the majority of the available probe-beam energy was discarded with the bandwidth, starving the CARS instrument of the probe-pulse energy required to generate sufficient signal in high-temperature environments. Flame thermometry via fs/ps rotational CARS was very recently demonstrated up to $T=1500 \mathrm{~K}$ by Dedic et al. [35], albeit by averaging of the signal over many laser shots.

To overcome the energy limitations of direct bandwidth removal for single-shot rotational spectroscopy in flames, we have recently demonstrated [18] a new approach for more efficient generation of high-energy, sub-10-ps, kHz-rate probe pulses based on second harmonic bandwidth compression (SHBC). SHBC is based on sum-frequency generation using broadband pulses with phase-conjugate linear chirps, and provides 5-6 ps probe pulses with energies in excess of $1 \mathrm{~mJ}$, greatly in exceeding the $4-20 \mu \mathrm{J}$ probe available energies in $[14,16,17,22,35]$. This SHBC approach enabled us to demonstrate single-shot thermometry and $\mathrm{O}_{2} / \mathrm{N}_{2}$ ratio measurements in $\mathrm{H}_{2}$ /air flat flames at temperatures as high as $T=2000 \mathrm{~K}$ [18].

In this paper, we systematically and quantitatively evaluate the performance of the SHBC fs/ps rotational CARS technique that was introduced in [18] over a limited range of flame 
conditions. A much wider range of temperature and stoichiometry is investigated, with both $\mathrm{H}_{2}$ and a hydrocarbon, $\mathrm{C}_{2} \mathrm{H}_{4}$, fuel. Two well-characterized canonical flat flame burners are selected, with near-adiabatic $\mathrm{H}_{2}$ /air flames investigated on the Hencken burner [44] for equivalence ratios $\phi=0.2$ to 1.2 , and premixed $\mathrm{C}_{2} \mathrm{H}_{4} /$ air flames investigated on the McKenna burner [45, 46] up to $\phi=3.14-$ a circumstance where nonresonant background, soot and an uncertain collisional environment often challenge CARS thermometry. Emphasis is placed on a quantitative assessment of the accuracy, and particularly the precision, of the measurements across this wide range of flame environments, putting the hybrid fs/ps rotational CARS diagnostic on sound quantitative footing as a combustion probe. We provide collision-free rotational CARS spectra for comparison to existing nanosecond CARS results that have employed extensive collisional modeling $[46,47]$ in the canonical McKenna burner system. The present work additionally provides a quantitative assessment of single-shot species measurements $\left(\mathrm{O}_{2} / \mathrm{N}_{2}\right)$ in flames using femtosecond CARS. Previous work [17, 35, 48-50] has provided demonstration of species detection in low-temperature gas cells and furnaces with well-defined mixtures, or qualitative species imaging $[51,52]$. This study extends the technique to quantitative concentration monitoring in flame environments with simultaneous temperature measurement.

\section{2. fs/ps Rotational CARS instrument}

A schematic of the fs/ps hybrid CARS instrument is shown in Figure 2. A commercial Ti:sapphire amplifier (Spectra Physics Solstice) provided nearly Fourier-transform-limited, 90fs duration, 3.1-mJ pulses at $1-\mathrm{kHz}$ repetition rate. The amplifier output spectrum was centered near $800 \mathrm{~nm}$, with a nominal bandwidth of $\sim 180 \mathrm{~cm}^{-1}$ (FWHM). Eighty percent of the amplifier output energy was used to generate a frequency-narrow probe pulse at $400 \mathrm{~nm}$, using the 
commercially available (Light Conversion SHBC) "Second-Harmonic Bandwidth Compressor" device illustrated in Figure 3, and described in detail below. The remaining twenty percent of the amplifier output was regulated with a variable attenuator and then split 50/50 to form the pump and Stokes pulses. The available pump/Stokes preparation bandwidth was optimized using the weak spectral focusing scheme described in [53], where small amounts of delay are introduced between the pump and Stokes pulses to shift the preparation bandwidth center by $\sim 100 \mathrm{~cm}^{-1}$ toward the anti-Stokes side of the CARS/CSRS spectrum. In this manner, signal generation for transitions in the $250-300 \mathrm{~cm}^{-1}$ range was enhanced by an order of magnitude [53]. Pump and Stokes energies were $\sim 50-90 \mu \mathrm{J} /$ pulse each for the $\mathrm{H}_{2} /$ air flame measurements, and up to 100 $\mu \mathrm{J} /$ pulse each for the $\mathrm{C}_{2} \mathrm{H}_{4} /$ air flame experiments. Probe pulse energy delivered to the measurement volume varied from $350-1100 \mu \mathrm{J} /$ pulse by variably aperturing the probe beam profile about its center.

Both folded and planar BOXCARS phase matching schemes [54] were employed with a 500$\mathrm{mm}$ focal length CARS beam-crossing lens to generate a signal beam near $400 \mathrm{~nm}$ that was sufficiently intense to be seen by eye in room-temperature air. The linear pump and probe polarizations were rotated to be orthogonal to the Stokes beam, so that the resulting CARS signal beam was orthogonally polarized to the high-energy pump, allowing for efficient rejection of intense probe-beam radiation with an analyzer placed in the CARS collection optics [55]. Dichroic mirrors separated the CARS signal from the $800-\mathrm{nm}$ preparation pulses, and additional probe background rejection was provided by careful placement of apertures in the signal collection path. The CARS spectra were dispersed onto a back-illuminated electron-multiplying CCD detector at $\sim 0.5 \mathrm{~cm}^{-1} /$ pixel using a 1 -m grating spectrograph with an $1800 \mathrm{l} / \mathrm{mm}$ grating. The detector was operated without electron-multiplication and with a gain setting of $\sim 1 \mathrm{e}^{-} /$count. 
Adaptation of the SHBC device for fs/ps rotational CARS measurements has been recently summarized [18], and additional detail is provided here. The SHBC utilizes principles first demonstrated by Raoult et al. [56]. As shown in the top portion of Figure 3, a broadband $~ 90-f s$ pump beam is split into two equal-energy legs. The femtosecond pumps are temporally stretched to several picoseconds using two carefully matched pulse stretchers. Each stretcher is essentially the front half of a Martinez-type " $4 f$ " design [57, 58], with identical unit-magnification cylindrical imaging telescopes and a common diffraction grating. A linear chirp, or time dependence of the center frequency, $\omega(t)$, of the stretched pump beams is introduced via differences in the relative path lengths of the red and blue spectral components. The amount of chirp added is controlled by perturbing the separation between the grating and the cylindrical lenses by distances of $\pm \delta$ about the lens focal length, $f$. As illustrated in the lower portion of Figure 3, with grating-to-lens separation $L_{1}=f-\delta$, the red components of the pump spectrum phase lead the blue, with the instantaneous frequency of the stretched picosecond beam given by $\omega_{1}(t+\Delta t)=\omega_{\mathrm{o}}+(\mathrm{d} \omega / \mathrm{d} t) \Delta t$. Similarly, when $L_{2}=f+\delta$, the blue components lead the red with equal and opposite (conjugate) phase, with $\omega_{2}(t+\Delta t)=\omega_{\mathrm{o}}-(\mathrm{d} \omega / \mathrm{d} t) \Delta t$. Sum-frequency generation (SFG) of the two pumps in beta-barium-borate $(\mathrm{BBO})$ results in annihilation of the linear chirps via $\omega_{\mathrm{SFG}}=\omega_{1}+\omega_{2}=2 \omega_{\mathrm{o}}$. The output beam is centered about the second harmonic of the initial 800-nm pump carrier frequency, with a narrow bandwidth that approaches the transform limit of the ps-duration chirped pump pulses. In these experiments, the 400-nm probe pulse-duration was $5.4 \mathrm{ps}$ and $5.8 \mathrm{ps} \mathrm{FWHM}$ for the $\mathrm{H}_{2} /$ air and $\mathrm{C}_{2} \mathrm{H}_{4}$ /air flame measurements, respectively, as determined by cross-correlation of the probe pulse with the femtosecondduration nonresonant CARS polarization in argon. Corresponding probe bandwidths were measured with a grating spectrograph to be $3.5 \mathrm{~cm}^{-1}$ and $4.1 \mathrm{~cm}^{-1}$, respectively. 


\section{Rotational fs/ps CARS processes and modeling}

In contrast to nanosecond CARS, which is treated as a steady process and modeled in the frequency domain, femtosecond CARS is modeled as a transient in the time domain $[14,36,37$, 59]. Broadband femtosecond-duration pump and Stokes pulses arrive nearly simultaneously and prepare a rotational polarization that decays in time as a result of collisional dephasing over $\sim 100$ ps at atmospheric pressure. Infinite bandwidth pump and Stokes pulses are assumed, so that these fields need not be included in the model. Nonresonant background in the flame measurements is eliminated with time-delayed probing, so we need only consider the Raman-resonant polarization, which is modeled as

$$
\chi(t)=\sum_{k} \sum_{v} \sum_{\Delta J= \pm 2} W_{v, J J^{\prime}}^{(k)} \exp \left[\left(i \omega_{v, J J^{\prime}}^{(k)}-\Gamma_{J J^{\prime}}^{(k)}\right) t\right]
$$

In Eq. 1, the sums are taken over all rotationally resonant species considered $(k)$, at thermally populated vibrational, $v$, and rotational, $J$, quantum levels. The $\omega_{v, J J^{\prime}}^{(k)}$ are the Raman frequencies, taken from the Sandia CARSFT code [60], for a pure-rotational $(\Delta v=0)$ transition in vibrational quantum level, $v$, from initial rotational quantum number $J$ to $J^{\prime}=J \pm 2 . \Gamma_{J J^{\prime}}^{(k)}$ are the Raman linewidths, which reflect the decay of $\chi$ as a result of collisional dephasing. The weight functions in Eq. 1 are given by

$$
W_{v, J J^{\prime}}^{(k)}=X_{k} \gamma_{v, k}^{2} b J J^{\prime} F_{J J^{\prime}}^{(k)}\left(N_{v, J^{\prime}}^{(k)}-N_{v, J}^{(k)}\right)
$$


In Eq. 2, the $N_{v, J}^{(k)}$ are the temperature-dependent rotational-vibrational Boltzmann fractions; $X_{k}$ are the species mole fractions; and $\gamma_{v, k}^{2}$ is the polarizability anisotropy of the $k$ th species in vibrational level, $v$, calculated using expressions given by Drake [61]. The $b_{J J^{\prime}}$ are the PlaczekTeller rotational line strength factors,

$$
b_{J, J+2}=\frac{3(J+1)(J+2)}{2(2 J+3)(2 J+1)}
$$

and

$$
b_{J, J-2}=\frac{3(J-1) J}{2(2 J-1)(2 J+1)}
$$

$F_{J J^{\prime}}^{(k)}$ are the Herman-Wallis factors, which modify the rotational line strength to include the effect of vibration-rotation interaction. These $J$-dependent corrections have recently been evaluated by Bohlin et al. [62] for rotational CARS, from which we use the expression recommended by Martinsson et al. [63],

$$
F_{J J^{\prime}}^{(k)}=1+4.448\left(\frac{2 B_{e}^{(k)}}{\omega_{e}^{(k)}}\right)^{2}\left(J^{2}+3 J+3\right)
$$

where $B_{e}$ and $\omega_{e}$ are the equilibrium molecular rotation and vibration constants for species $k$, respectively.

Upon introducing a probe pulse at delay, $\tau$, the CARS electric field is calculated from, 


$$
E_{C A R S}(t-\tau)=\chi(t) \sqrt{I_{p r}(t-\tau)}
$$

where $I_{p r}$ is the intensity pulse shape of the assumed-transform-limited probe, as measured by cross-correlation. The squared amplitude of the Fourier transform of the field in Eq. 6 yields the CARS spectrum, which is convolved with a Gaussian instrument function for least-squares fitting of the experimental data using a standard nonlinear optimization routine. Experimental spectra were background subtracted for stray light, normalized by nonresonant CARS spectra recorded in argon at $\tau=0$ to account for the finite bandwidth of the pump and Stokes pulses, and finally normalized to unit maximum for fitting. Temperature and species concentrations relative to $\mathrm{N}_{2}$ were fitting parameters, along with wavenumber axis shifting and stretching parameters and an intensity offset. The probe pulse delay was also permitted to vary in the fitting within a limited \pm 0.5 -ps range around the estimated value from the experiment.

The result for $\chi$ is purely imaginary and is plotted at room temperature for four different molecules of interest in hydrocarbon/air combustion in Figure 4. For each species, the timedomain response is characterized by well-defined recurrence peaks, with a period that is inversely proportional to the well-defined $\sim 4 B_{e}$ spacing between the lines in the respective rotational Raman frequency spectra [22]. For the analysis of the $\mathrm{H}_{2} /$ air and lean $\mathrm{C}_{2} \mathrm{H}_{4} /$ air flames reported here, $\mathrm{N}_{2}$ and $\mathrm{O}_{2}$ are included in the calculations. $\mathrm{CO}$ is additionally available in the model for application to fuel-rich hydrocarbon/air flames. The response for $\mathrm{CO}_{2}$ in the lowest lying vibrational state is additionally included in Figure 4, alongside the measured probe pulse shape at a delay of $\tau=16 \mathrm{ps}$, to illustrate the selection of probe-beam delay for our measurements in $\mathrm{C}_{2} \mathrm{H}_{4} /$ air flames. $\mathrm{CO}_{2}$ can contribute significantly to the rotational Raman spectrum in fuel-rich flames, and the 16-ps delay is selected so that the probe pulse arrived in 
between recurrences in the $\mathrm{CO}_{2}$ response. Contributions from $\mathrm{CO}_{2}$ that can complicate the Raman spectrum below $100 \mathrm{~cm}^{-1}$ [42] are minimized, and $\mathrm{CO}_{2}$ was neglected in the spectral fitting routine, greatly facilitating speed. Selection of probe pulse delay was less critical in the $\mathrm{H}_{2} /$ air flame experiments and was set to $\tau=11.5 \mathrm{ps}$ there.

\section{Results and discussion}

\subsection{Hydrogen/air flames stabilized on the Hencken burner}

Rotational CARS measurements were performed in nearly adiabatic $\mathrm{H}_{2}$ /air flames stabilized on a $50-\mathrm{mm}$ square Hencken burner for equivalence ratios $\phi=0.2$ to 1.2 . The air-flow rate was fixed at 85.2 SLPM and fuel flow rate monotonically varied from 7 to 42 SLPM. Gas flows were metered with thermal-type mass flow controllers (MKS "M series"). Manufacturer-specified accuracy in the metered gas flow rates was $\pm 2 \%$ of full scale, or \pm 1 SLPM for $\mathrm{H}_{2}$ and \pm 2 SLPM for air, which propagated into uncertainties (95\% confidence) in $\phi$ ranging from a maximum of $\pm 14 \%$ at $\phi=0.2$ that decreased to $\pm 8 \%$ at $\phi=0.3 ; \pm 5.2 \%$ at $\phi=0.6$; and $\pm 3.5 \%$ at $\phi=1.2$.

Spectra obtained in $\mathrm{H}_{2}$ /air flames are presented alongside best-fit results for six different equivalence ratios in Figure 5. Both single-laser-shot spectra (right-hand side) and data that have been averaged for 1000 laser shots (left-hand) are shown in order to highlight the repeatability of the single-shot data. Best-fit temperature and $\mathrm{O}_{2} / \mathrm{N}_{2}$ ratio are indicated on the plots for reference. The quality of the theoretical fits shown in the Figure is quite good in all cases over a wide range of temperatures from $\sim 1240 \mathrm{~K}$ to $2375 \mathrm{~K}$. Under these flame conditions, and at the $\sim 3.5 \mathrm{~cm}^{-1}$ probe bandwidth employed, contributions from $\mathrm{N}_{2}$ and $\mathrm{O}_{2}$ rotational lines cannot generally be separated. The spectra are dominated by contributions from $\mathrm{N}_{2}$, with the impact of $\mathrm{O}_{2}$ realized as an alteration of the spectral envelope formed by the peak intensity profile and some frequency 
shifting of select lines. For comparison, the high-temperature spectra at the bottom of Figure 5 for $\phi=1.12$ are essentially devoid of $\mathrm{O}_{2}$ content and exhibit the smooth intensity envelope typically observed nanosecond rotational CARS spectra of $\mathrm{N}_{2}$.

Accuracy of the fitted temperatures and concentration ratios was assessed by comparison to adiabatic equilibrium calculations. The results are shown in Figure 6a, where data from two different experimental campaigns taken over a year apart are presented. Fitted temperatures and $\mathrm{O}_{2} / \mathrm{N}_{2}$ ratios are represented by red and blue symbols, respectively, with equilibrium calculations shown as solid red and blue lines. Results obtained from fits to a single spectrum averaged for 1000 laser shots are represented by filled symbols, while data representing the mean of 1000 single-laser-shot realizations acquired at $1 \mathrm{kHz}$ are indicated by open symbols. Equilibrium temperature uncertainty as a result of the metered gas-flow rates ranges from $\pm 70 \mathrm{~K}(5.8 \%)$ down to $\pm 10 \mathrm{~K}(<1 \%)$ as $\phi$ was varied from 0.3 to 1.2 . Uncertainty in calculated $\mathrm{O}_{2} / \mathrm{N}_{2}$ ratio was essentially constant near \pm 0.008 for $\phi$ up to 0.8 and then decreased monotonically to zero at $\phi \geq$ 1, where no oxygen is present in the product gases. Equivalence ratios for data set 1 were fixed at the metered levels, while the $\phi$ axis was shifted by +0.05 for data set 2 to best match the shape of the measured curve to equilibrium, a common practice for Hencken-burner measurements [44], where the measured shape of the equilibrium temperature curve is likely a better indicator of $\phi$ than the metered gas flows. Because error in the metered flow rates was dominated by their accuracy, and not their precision or stability, and because the measurements were additionally conducted with the air flow held fixed and the fuel-flow monotonically adjusted, the resulting error in the equivalence ratio data was expected to primarily result in a systematic bias, justifying a global shift of this type. In addition, the measured temperatures do not exhibit a significant 
low-temperature bias, so that heat losses to the burner surface likely result in temperature changes that are less than the observed measurement accuracy.

For $\phi \geq 0.3$, measured temperatures are generally within $\pm 5 \%$ of equilibrium values, with roughly $70 \%$ of the results within $\pm 3 \%$. The two data points for $\phi<0.3(T<1150 \mathrm{~K})$ appear to be outliers, where the temperature becomes systematically lower than equilibrium up to $-10 \%$ at $\phi=0.2$. The $\mathrm{O}_{2} / \mathrm{N}_{2}$ ratio additionally becomes systematically higher than equilibrium at $\phi \leq 0.3$ as well. The cause for these outliers is not understood. Earlier measurements using a similar fs/ps rotational CARS scheme in $\mathrm{N}_{2}$ near $T=500 \mathrm{~K}$ [22] and in furnace-heated air up to $T=800 \mathrm{~K}$ [17] indicated an accuracy of $2.5 \%$ or better in the temperature data, so that large systematic errors at low temperature should not be expected in these measurements.

Measured $\mathrm{O}_{2} / \mathrm{N}_{2}$ ratios are generally within $\pm 5-10 \%$ of equilibrium for $\mathrm{O}_{2} / \mathrm{N}_{2}$ of $6 \%$ or more. At lower $\mathrm{O}_{2} / \mathrm{N}_{2}$ levels - absolute $\mathrm{O}_{2}$ mole fraction of $4 \%$ or less-oxygen sensitivity in the spectra becomes less significant, the measurement accuracy degrades to $\pm 20-40 \%$, which is amplified in part by the low absolute levels of oxygen as $\mathrm{O}_{2} / \mathrm{N}_{2}$ approaches zero. The impact of uncertainty in the metered gas flow rates can be minimized by replotting both the measurements and equilibrium calculations as temperature vs. $\mathrm{O}_{2} / \mathrm{N}_{2}$ ratio, as shown in Figure $6 \mathrm{~b}$. When this is done, over $90 \%$ of the simultaneous temperature/oxygen measurements fall within $\pm 5 \%$ of the equilibrium curve, and $75 \%$ of the data are within $\pm 3 \%$. This $\pm 3-5 \%$ level of accuracy over a wide range (1000-2400 K) of temperatures is comparable to values reported for nanosecond rotational CARS in furnace-heated air and $\mathrm{O}_{2} / \mathrm{N}_{2}$ mixtures for temperatures as high as $2050 \mathrm{~K}$ $[38,40,64]$, and slightly worse than the $2.5 \%$ levels for fs/ps rotational CARS in air below 800 $\mathrm{K}[17]$. 
The precision of the single-laser-shot data was investigated by fitting ensembles of 1,000 single-shot spectra acquired at $1-\mathrm{kHz}$ data rate. Histograms of single-shot temperature and $\mathrm{O}_{2} / \mathrm{N}_{2}$ ratio are shown in Figure 7 for lean $\mathrm{H}_{2} /$ air flames with $\phi=0.20$ to 0.89 . Here, we can see that the temperature-measurement precision, as quantified by a single standard deviation as a fraction of the mean, $\sigma_{T} / \bar{T}$, is $1-1.5 \%$ for lean flames at temperatures up to $T=1550 \mathrm{~K}(\phi=0.44)$. This temperature is close to the crossover value identified by Seeger and Leipertz [38], below which rotational CARS is a more effective thermometer than vibrational CARS. At $1-1.5 \%$, this thermometric precision is a $\sim 3-4 \times$ improvement over reported values for nanosecond rotational CARS $[38,40,64]$, acquired under furnace conditions. Moreover, the $1-1.5 \%$ thermometric precision observed here is comparable the best reported fs vibrational CARS measurements in flames by Miller et al. [12] at 2\%, and Richardson et al. [24] at 0.7-1\%, and is comparable to or better than reported precision using fs rotational CARS at low temperatures: including our results [17] in furnace-heated air up to $T=800 \mathrm{~K}(0.8-2 \%)$; data from Stauffer et al. [22] in pure $\mathrm{N}_{2}$ at $T=540 \mathrm{~K}(2.5 \%)$; and room-temperature $\mathrm{N}_{2}$ results (1.4\%) by Bohlin and Kliewer [19]. Above $1550 \mathrm{~K}$, the thermometric precision degrades, but remains at 3-4\% levels, comparable to the best reported results for nanosecond rotational CARS in controlled oven and furnace environments up to $T \sim 2200 \mathrm{~K}[38,40,64]$. The precision of the relative oxygen concentration measurement is similar to thermometric values, at $\sigma_{\mathrm{O}_{2}} / \overline{\mathrm{O}_{2}}=1-2 \%$ up to $\phi=0.44$, where $T=1550 \mathrm{~K}$-a level which exceeds the $\sim 6 \%$ relative precisions reported for nanosecond rotational CARS [38, 40, 64]. At more rich conditions and higher temperatures, $\sigma_{\mathrm{O}_{2}} / \overline{\mathrm{O}_{2}}$ systematically increases, $\overline{\mathrm{O}_{2}}$ becomes very low, and the width of the histograms in Figure 7 spreads.

As a demonstration of high-precision real-time monitoring of low-amplitude flame dynamics, fluctuations in temperature and stoichiometry were monitored in the Hencken burner product 
gases. In early experiments, flame instabilities were observed as a result of a faulty check valve in the fuel flow system, resulting in an audible "hum" emanating from the burner under very lean conditions. Time series temperature/oxygen data acquired at $\phi=0.34$ and a rate of $1 \mathrm{kHz}$ are shown in Figure 8a, and the associated power spectrum of the temperature signal is shown in Figure $8 \mathrm{~b}$. An oscillation in the temperature signal with $\sim 30 \mathrm{~K}$ amplitude and $\sim 4$ ms period is readily distinguishable. Periodic fluctuations in the temperature and $\mathrm{O}_{2} / \mathrm{N}_{2}$ levels display a strong, negative correlation, which is consistent with oscillations in the flame stoichiometry. Inspection of the power spectrum reveals a distinct peak at $f=232 \mathrm{~Hz}-\mathrm{a}$ frequency that is consistent with the pitch of audible sound emitted from the flame. Detection of these lowamplitude instabilities, of only $2.4 \%$ in temperature and $1.5 \%$ in $\mathrm{O}_{2}$, is made possible by the very high precision offered by the fs/ps CARS technique.

\subsection{Ethylene/air flames stabilized on the McKenna burner}

A water-cooled sintered bronze McKenna burner was used to investigate the performance of the fs/ps rotational CARS technique in a more complex hydrocarbon-fueled flame. This canonical burner is especially relevant because it has been selected to provide benchmark sooting flames by both the international laser-induced incandescence (LII) and sooting flame workshops $[65,66]$. The total flow rate of $\mathrm{C}_{2} \mathrm{H}_{4}$ and air was held constant at 10 SLPM and no shroud gas flow was provided. A 12.7-mm-thick, 60-mm-diameter steel stabilization plate was positioned 30 $\mathrm{mm}$ above the burner surface to minimize buoyancy driven unsteadiness in these flat flames. Spectra were acquired at equivalence ratios of $\phi=0.75,0.91,1.58,2.51$ and 3.14. Accuracies of the metered gas flow rates were \pm 0.06 SLPM for $\mathrm{C}_{2} \mathrm{H}_{4}$ and \pm 0.15 SLPM for air, which resulted 
in uncertainties in $\phi$ that ranged from a worst case of $\pm 12 \%$ at $\phi=0.75$ down to $\pm 3.8 \%$ at $\phi=$ 3.14 .

Hydrocarbon flames have traditionally presented a challenging environment for CARS diagnostics using nanosecond laser pulses. CARS spectra often exhibit significant nonresonant background interference, particularly under fuel-rich conditions [28], and broadening of the dominant $\mathrm{N}_{2}$ and $\mathrm{O}_{2}$ lines from collisions with multiple species, including $\mathrm{H}_{2} \mathrm{O}, \mathrm{CO}, \mathrm{CO}_{2}$, and $\mathrm{H}_{2}$ must be carefully taken into account $[46,67]$. Rotational CARS spectra acquired at three different probe delays in the product gases of a $\phi=0.75 \mathrm{C}_{2} \mathrm{H}_{4} /$ air flame at a height of $z=11.5$ $\mathrm{mm}$ above the burner are presented in Figure 9. At a probe delay of $\tau=0 \mathrm{ps}$, a strong nonresonant background contribution to the signal is observed, which is completely eliminated at delays of $\tau=16 \mathrm{ps}$ and $21.5 \mathrm{ps}$, where there is no temporal overlap of the probe and preparation pulses. At $\tau=21.5 \mathrm{ps}$, the probe pulse is coincident with the $\mathrm{CO}_{2}$ recurrence peak shown in Figure 4, and a strong contribution from $\mathrm{CO}_{2}$ dominates the spectrum for $\omega<100 \mathrm{~cm}^{-1}$. At $\tau=$ $16 \mathrm{ps}$, contributions from $\mathrm{CO}_{2}$ to the spectrum at $\omega<100 \mathrm{~cm}^{-1}$ have been minimized, but not entirely eliminated, because the tails of the probe pulse profile still overlap $\mathrm{CO}_{2}$ rotational recurrence peaks shown in Figure 4. A shorter duration probe could completely "turn off" the $\mathrm{CO}_{2}$ contribution if desired.

Shot-averaged and single-laser-shot spectra acquired in the product gases of multiple $\mathrm{C}_{2} \mathrm{H}_{4} /$ air flames with the probe delay fixed at $\tau=16 \mathrm{ps}$ are shown in Figure 10. The spectra were acquired at $z=11.5 \mathrm{~mm}$ above the burner surface, with the exception of the shot-averaged spectrum at $\phi=3.14$, which was acquired at $z=18 \mathrm{~mm}$ where the soot volume fraction was $f_{v}=$ $0.3 \mathrm{ppm}$ [68] to illustrate the performance of the technique with significant soot in the 
measurement volume. Under fuel-lean conditions, the spectra display contributions from both $\mathrm{O}_{2}$ and $\mathrm{N}_{2}$ and the fits are of similar quality to the results shown above for $\mathrm{H}_{2} /$ air combustion, with the exception of some discrepancy at $\omega<100 \mathrm{~cm}^{-1}$ arising from residual $\mathrm{CO}_{2}$ contributions to the spectra, which were not modeled here. At $\phi=0.75$, the measured $\mathrm{O}_{2} / \mathrm{N}_{2}$ of $6.6 \%$ is in excellent agreement with the equilibrium value of $6.4 \%$, which should not be greatly affected by heat transfer from this non-adiabatic flame [46]. At $\phi=0.91$, contributions from $\mathrm{O}_{2}$ are near zero, and the spectrum exhibits a smooth $\mathrm{N}_{2}$ intensity envelope.

In rich flames for $\phi \geq 1.58$, the $\mathrm{CO}_{2}$ contribution is greatly diminished, improving the quality of the fits at low Raman frequencies. However, the $\mathrm{N}_{2}$ spectral intensity envelope is perturbed, with some systematic deviation between the measured and calculated spectra apparent for select $\mathrm{N}_{2}$ lines in the vicinity of $\omega=180-200 \mathrm{~cm}^{-1}$ and $\omega=250 \mathrm{~cm}^{-1}$. This discrepancy becomes greater as $\phi$ is increased to 2.51 and 3.14. The reason for this deviation between theory and experiment is not completely understood, but is presumed to be an effect of $\mathrm{CO}$ on the $\mathrm{N}_{2}$ spectrum. CO concentrations increase markedly at these very rich conditions, and $\mathrm{CO}$ was added to the spectral modeling code of Eqs. 1-6, for fitting of data acquired in fuel-rich flames. Inclusion of $\mathrm{CO}$ improved the quality of the fits and the evaluated temperatures, but $\mathrm{CO} / \mathrm{N}_{2}$ levels were dramatically underpredicted relative to expected levels for this flame [46] and are not presented here.

Heat losses from the water-cooled McKenna burner prohibit a valid comparison of CARSmeasured temperatures with adiabatic equilibrium, as was performed in the case of $\mathrm{H}_{2} /$ air flat flames above. Instead, the new fs/ps rotational CARS data were benchmarked against vertical temperature profiles measured with nanosecond rotational CARS and dual-pump vibrational 
CARS [68] in our laboratory, and against a single-point nanosecond rotational CARS measurement obtained at Lund University [46]. Both nanosecond rotational CARS systems utilized the dual-broadband configuration [69] based on a frequency doubled Nd:YAG laser and broadband dye-laser employing DCM dye. Our nanosecond CARS data were analyzed using the Sandia CARSFT code [60] and considering self-broadened linewidths. Vestin et al. [46] fitted their nanosecond rotational CARS spectra by considering both $\mathrm{N}_{2}-\mathrm{N}_{2}$ self-broadening, and a more detailed collisional model that included $\mathrm{N}_{2}$ lines broadened by $\mathrm{N}_{2}, \mathrm{CO}, \mathrm{CO}_{2}$, and $\mathrm{H}_{2} \mathrm{O}$ collisions. Bohlin [47] reconsidered Vestin's spectra by including the effect of $\mathrm{N}_{2}-\mathrm{H}_{2}$ collisions, so that all major product-gas species were included in his analysis. In contrast, the fs/ps CARS spectra for $\tau=16 \mathrm{ps}$ are essentially collision-free, eliminating the need for detailed linewidth modeling. To confirm this, the shot-averaged spectra presented in Figure 10 were fit by neglecting collisional dephasing by setting $\Gamma=0$ in Eq. 1, by using $\mathrm{N}_{2}-\mathrm{N}_{2}$ self-broadened linewidths, and by using Bohlin's model [47] that accounted for all major collisional partners. Inclusion of collisions resulted in a maximum change of $0.75 \%$, or only $8-11 \mathrm{~K}$ in the evaluated temperature, with this maximum deviation occurring for $\mathrm{N}_{2}-\mathrm{N}_{2}$ self-broadening.

Temperature profiles obtained at fuel-rich conditions with $\phi=1.58,2.51$, and 3.14 are shown in Figure 11, where the quantitative agreement of temperatures measured with the new fs/ps rotational CARS scheme and the benchmark nanosecond CARS data is quite good. Temperatures from all three interpretations of Vestin's spectra are included in Figure 11. Differences in collisional models result in a $\sim 3.5-4 \%$ spread in the temperatures evaluated from Vestin's spectra. In the stable portion of the flame, for $4 \mathrm{~mm} \leq z \leq 15 \mathrm{~mm}$, fs/ps CARS temperatures are generally within $1 \%$ of nanosecond results at $\phi=1.58$ and, and within $3 \%$ at $\phi=2.51$ and 3.14 . This level of agreement is remarkable when one considers that the nanosecond- and fs/ps-CARS 
measurements were taken weeks to years apart, with both rotational and vibrational spectroscopies, and in two different McKenna burners in separate laboratories. Best agreement of the collision-free fs/ps results with Vestin's single-point measurements is achieved when $\mathrm{N}_{2}-\mathrm{H}_{2}$ collisions are omitted from the model. Uncertainties associated with linewidth data may be approaching other sources of error in the measurement, such as time-dependent shifts in the spectrum of the broadband pump/Stokes source (as measured by nonresonant CARS in argon).

As in the case of the $\mathrm{H}_{2} /$ air flames above, the precision of the temperature measurements in these hydrocarbon flames was investigated using ensembles of 1000 single-laser-shot measurements. Temperature histograms acquired at four equivalence ratios in the range $\phi=0.75$ to 3.14 are shown in Figure 12. At these particular values of $\phi$, the temperature varies by only $\sim 8 \%$, and the variation in CARS signal strength results mainly from a decrease in $\mathrm{N}_{2}$ as the flames becomes richer. The impact of signal-to-noise on precision can then be investigated nearly independently of the temperature sensitivity of the rotational spectroscopy employed. At $\phi$ $=0.75, \sigma_{T} / \bar{T}$ is better than $1 \%$, which is comparable to the best reported results with femtosecond CARS over a wide range of temperatures $[12,17,24]$. As $\phi$ is increased, the peak CARS signal strength decays by more than $4 \times$ and the precision degrades to $3.1 \%$ by $\phi=3.14-$ a value that is still on par with the best nanosecond rotational CARS measurements [38, 40, 70].

\subsection{Relationship between precision and signal-to-noise ratio}

The impact of CARS signal strength on measurement precision was investigated by plotting $\sigma_{T} / \bar{T}$ and $\sigma_{\mathrm{O} 2} / \overline{\mathrm{O}_{2}}$ vs. peak signal-to-noise ratio (SNR), as determined from detector readoutnoise and photon shot-noise contributions, 


$$
S N R=N_{e^{-}} / \sqrt{R^{2}+N_{e^{-}}},
$$

where $N_{e^{-}}$is the peak recorded photoelectron count in the spectrum and $R$ is the measured detector readout noise of $11 \mathrm{e}^{-}$(rms). Results from both the $\mathrm{H}_{2} /$ air and $\mathrm{C}_{2} \mathrm{H}_{4} /$ air flame measurements are plotted in Figure 13, where the precision, $\sigma_{T} / \bar{T}$, is observed to hover between 1 and $1.5 \%$ over a wide range of temperatures for peak SNR in excess of $\sim 60$, with $\sigma_{\mathrm{O} 2} / \overline{\mathrm{O}_{2}}$ values of 1-2\% observed in this same range of SNR. The results for the $\mathrm{C}_{2} \mathrm{H}_{4}$ McKenna flames, acquired over a limited $8 \%$ change in temperature, are plotted in blue and fall in close agreement with the data from the $\mathrm{H}_{2} /$ air flames over a much broader range of conditions. This critical SNR of 60 is likely dependent on system parameters, in particular the bandwidth of the femtosecond pump/Stokes source, but the observation of a critical SNR for high-precision measurements over a wide range of flame conditions is intriguing. It is well-known that rotational CARS spectra exhibit reduced temperature sensitivity at high temperatures [39], and additional measurements would be required to determine if an upper temperature limit exists where the potentially reduced high-temperature sensitivity of rotational Raman spectroscopy begins to ultimately degrade the measurement precision, as opposed to CARS signal photons and SNR.

\section{Summary and Conclusions}

The performance of a recently demonstrated [18] hybrid fs/ps rotational CARS scheme that employs SHBC-generated high-energy, sub-10-ps probe pulses for kHz-rate measurements has been critically assessed. These short-duration, high-energy SHBC pulses permit nonresonantbackground- and collision-free spectra with sufficient CARS photon yields for single-shot measurements in flames. The system performance has been characterized for temperature and 
relative $\mathrm{O}_{2} / \mathrm{N}_{2}$ concentration measurements in the product gases of two canonical flat flames: a near-adiabatic $\mathrm{H}_{2}$ /air flame stabilized on the Hencken burner; and a premixed $\mathrm{C}_{2} \mathrm{H}_{4}$ /air flame on the McKenna burner. Our previous demonstration of the technique has been extended to a much wider range of flame conditions, with temperatures varying from $T=800 \mathrm{~K}$ to $2400 \mathrm{~K}, \mathrm{O}_{2}$ content from 0 to $\sim 16 \%$ absolute, and from clean $\mathrm{H}_{2}$ combustion to a sooting, fuel-rich hydrocarbon flame.

Shot-averaged and single-laser-shot spectra are well-fit by a simple phenomenological model. In the $\mathrm{H}_{2}$ /air system, temperature measurements derived from fs/ps rotational CARS spectra are generally within $3-5 \%$ of adiabatic equilibrium values, while $\mathrm{O}_{2} / \mathrm{N}_{2}$ ratios within are within $5-10 \%$ of equilibrium for $\mathrm{O}_{2} / \mathrm{N}_{2}>\sim 6 \%$. Collision-free fs/ps rotational CARS temperatures are within $1-3 \%$ of nanosecond rotational and vibrational CARS measurements in the $\mathrm{C}_{2} \mathrm{H}_{4}$ /air flames - a level of agreement that is comparable to the variability in temperature due to collisional modeling $[46,47]$ in the analysis of nanosecond CARS spectra in hydrocarbon flames, and well within reported levels of accuracy of for nanosecond rotational CARS thermometry [38, 40, 64] under well-controlled furnace conditions with known collisional environments. Some systematic deviation at three select rotational lines was observed in the fits to spectra measured in rich $\mathrm{C}_{2} \mathrm{H}_{4} /$ air flames. At present, this error is presumed to be a result of the interaction of $\mathrm{CO}$ with the probe-beam electric field and the dominant $\mathrm{N}_{2}$ contribution to the spectrum, and does not seem to significantly impact the accuracy of the measured temperatures, which are mainly sensitive to the total spectral envelope formed by the rotational peaks.

The precision offered by low-noise femtosecond preparation of the rotational coherence is outstanding for both the temperature and $\mathrm{O}_{2} / \mathrm{N}_{2}$ ratio measurements in both the $\mathrm{H}_{2} /$ air and hydrocarbon/air systems. For peak SNR in excess of 60, attained for temperatures up to $T=1660$ 
$\mathrm{K}$ in $\mathrm{C}_{2} \mathrm{H}_{4}$ /air flames and $T=1550 \mathrm{~K}$ in $\mathrm{H}_{2}$ /air flames, temperature-measurement precision is $1-1.5 \%$, exceeding the best nanosecond rotational CARS measurements $[38,40]$ at atmospheric pressure by $\sim 3-4 \times$. At $1-1.5 \%$ the thermometric precision of current measurements is additionally comparable to the best reported results using femtosecond vibrational CARS schemes at flame temperatures exceeding $2000 \mathrm{~K}[12,24]$, and meets or exceeds the precision demonstrated using rotational fs/ps CARS in low-temperature environments in both air [17] and pure $\mathrm{N}_{2}[19,22]$. Precision of the $\mathrm{O}_{2}$ measurements is similar, at $1-2 \%$ at temperatures up to $T=$ $1550 \mathrm{~K}$ in lean $\mathrm{H}_{2}$ /air flames where similar SNR of 60 or more is achieved. The $\mathrm{kHz}$ repetition rate and high precision offered by the fs/ps CARS instrument has been demonstrated in an unstable $\mathrm{H}_{2}$ /air flame, where periodic temperature fluctuations at $\sim 230 \mathrm{~Hz}$, with an amplitude of only $\sim 30 \mathrm{~K}$ were monitored, and confirmed by anti-correlated low-amplitude oscillations in $\mathrm{O}_{2}$ content.

The technique has additional potential for multi-species monitoring with the addition of $\mathrm{CO}_{2}$ modeling, improvements in the modeling of the $\mathrm{CO}$ contribution, and the potential addition of $\mathrm{H}_{2}$ with more femtosecond bandwidth. $\mathrm{CO}_{2}$ signatures are observed to be particularly strong with this technique, and can be optimized with careful selection of the probe pulse delay.

\section{Acknowledgments}

The author thanks Alexis Bohlin, presently at Sandia, for providing the nanosecond rotational CARS results and linewidth data compiled at Lund University. Technical assistance from Tom Grasser is also gratefully acknowledged. Funding for this work has been provided by the United States Department of Energy through Sandia National Laboratories. Sandia is a multiprogram laboratory operated by Sandia Corporation, a Lockheed-Martin Company, for the United States 
Department of Energy's National Nuclear Security Administration under Contract DE-AC0494AL85000. 


\section{List of Figures}

Figure 1 (color online) - Energy level diagram for the fs/ps rotational CARS process: broadband femtosecond preparation pulses centered near $800 \mathrm{~nm}$, and represented by thick red lines, prepare a Raman coherence at time $t=0$. A frequency-narrow picosecond probe centered near $400 \mathrm{~nm}$, and shown as a narrow blue line, probes the coherence at time delay $t=\tau$, to yield a CARS signal shown as a thick violet line.

Figure 2 (color online) - Schematic of key elements of the fs/ps rotational CARS instrument: aperture $(\mathrm{A})$; polarizer $(\mathrm{PZ})$; periscope $(\mathrm{P})$; beam-expanding telescope $(\mathrm{T})$; half waveplate $(\lambda / 2)$. Computer controlled delay lines are indicated by dashed boxes with a double arrow.

Figure 3 (color online) - Second-harmonic bandwidth compression concept for high-energy probe beam generation. Major elements of the optical design where phase-conjugate linear temporal chirps are imparted to initially compressed femtosecond pump beams are shown at top: grating $(\mathrm{G})$; cylindrical lens (CL); doubling crystal (BBO). Temporal variation in the instantaneous frequencies of the chirped pump beams in the sum-frequency process (bottom).

Figure 4 (color online) - Time-domain picture of the hybrid fs/ps process. Calculated transient molecular Raman response functions of $\mathrm{N}_{2}, \mathrm{O}_{2}, \mathrm{CO}$, and $\mathrm{CO}_{2}$ are shown, with the measured probe-beam intensity at delay of $\tau=16$ ps plotted with the $\mathrm{CO}_{2}$ response.

Figure 5 (color online) - fs/ps rotational CARS spectra obtained in the products of nearadiabatic $\mathrm{H}_{2}$ /air flames stabilized on the Hencken burner. Equivalence ratios and fitted temperature and $\mathrm{O}_{2} / \mathrm{N}_{2}$ ratios are indicated on the plots. Shot-averaged (1000 shots) are shown in the left-hand column, while single-shot spectra are shown in the right-hand column.

Figure 6 (color online) - Temperature and $\mathrm{O}_{2} / \mathrm{N}_{2}$ ratios evaluated in the product gas of nearadiabatic $\mathrm{H}_{2}$ /air flames stabilized on the Hencken burner. Results from fits to both shot-averaged and single-laser-shot spectra are shown alongside adiabatic equilibrium calculations.

Figure 7 (color online) - Temperature (top) and $\mathrm{O}_{2} / \mathrm{N}_{2}$ ratio (bottom) histograms constructed from 1000 single-laser-shot fs/ps rotational CARS spectra obtained in the products of nearadiabatic $\mathrm{H}_{2}$ /air flames stabilized on the Hencken burner.

Figure 8 (color online) - Temperature and $\mathrm{O}_{2} / \mathrm{N}_{2}$ time series obtained at $1-\mathrm{kHz}$ data rate in a $\mathrm{H}_{2}$ /air flame stabilized on the Hencken burner at an equivalence ratio of $\phi=0.34$ (a). Power spectrum of the temperature data revealing a distinct peak at $f=232 \mathrm{~Hz}$ (b).

Figure 9 (color online) - Transient CARS spectra measured in a $\phi=0.75$ premixed $\mathrm{C}_{2} \mathrm{H}_{4} /$ air flame for different probe-beam delays, as indicated on the plots. At $\tau=0$, a strong nonresonant background contribution is observed. The nonresonant polarization has decayed to zero by $\tau=16$ 
ps, where the fs/ps CARS data were recorded. At $\tau=21.5 \mathrm{ps,} \mathrm{a} \mathrm{strong} \mathrm{contribution} \mathrm{from} \mathrm{CO}_{2}$ is observed at low Raman shifts.

Figure 10 (color online) - fs/ps rotational CARS spectra obtained in the products of $\mathrm{C}_{2} \mathrm{H}_{4} /$ air flames stabilized on the McKenna burner. Equivalence ratios and fitted temperature and $\mathrm{O}_{2} / \mathrm{N}_{2}$ ratios are indicated on the plots. Shot-averaged (1000 shots) are shown in the left-hand column, while single-shot spectra are shown in the right-hand column. All spectra were obtained at a height of $z=11.5 \mathrm{~mm}$ above the burner surface, with the exception of the shot-averaged spectrum at $\mathrm{f}=3.14$, which was acquired at $z=18 \mathrm{~mm}$ to illustrate performance under sooting conditions.

Figure 11 (color online) - Vertical temperature profiles obtained in the product gases of fuelrich $\mathrm{C}_{2} \mathrm{H}_{4}$ /air flames stabilized on the McKenna burner at equivalence ratios of $\phi=1.58,2.51$, and 3.14. Temperatures obtained from fits to shot-averaged fs/ps rotational CARS spectra are shown as blue circles. Temperatures measured with nanosecond CARS are included for comparison: ns rotational CARS measurements conducted at Sandia in Nov. 2013 (red triangles); vibrational dual-pump CARS measurements conducted at Sandia in 2006 [68] (green circles); single-point measurements from Lund $[46,47]$ and interpreted for three different sets of collider gases with $\mathrm{N}_{2}$ (green and white squares).

Figure 12 - Histograms constructed from 1000 single-laser-shot fs/ps rotational CARS temperature measurements at fuel-air equivalence ratios of $\phi=0.75,1.58,2.51$, and 3.14.

Figure 13 (color online) - Measurement precision in both temperature and $\mathrm{O}_{2} / \mathrm{N}_{2}$ ratio as a function of peak signal-to-noise ratio. 


\section{References}

1. S. Roy, J. R. Gord, A. K. Patnaik, Prog. Energy. Combust. Sci. 36 (2010) 280-306.

2. S. P. Kearney, K. Frederickson, T. W. Grasser, Proc. Combust. Inst. 32 (2009) 871-878.

3. C. J. Kliewer, Y. Gao, T. Seeger, J. Kiefer, B. D. Patterson, T. B. Settersten, Proc. Combust. Inst. 33 (2011) 831-838.

4. M. Aldén, S. Wallin, Appl. Opt. 24 (1985) 3434-3437.

5. A. C. Eckbreth, G. M. Dobbs, J. H. Stufflebeam, P. A. Tellex, Appl. Opt. 23 (1984) 13281339.

6. P. E. Bengtsson, M. Aldén, S. Kröll, D. Nilsson, Combust. Flame 82 (1990) 199-210.

7. E. J. Beiting, Appl. Opt. 25 (1986) 1684-1692.

8. R. P. Lucht, S. Roy, T. R. Meyer, J. R. Gord, Appl. Phys. Lett. 89 (2006) 251112.

9. $\quad$ R. P. Lucht, P. J. Kinnius, S. Roy, J. R. Gord, J. Chem. Phys. 127 (2007) 044316.

10. S. Roy, W. D. Kulatilika, D. R. Richardson, R. P. Lucht, J. R. Gord, Opt. Lett. 34 (2009) 3587-3589.

11. S. Roy, P. Wrzesinski, D. Pestov, T. Gunaratne, M. Dantus, J. R. Gord, Appl. Phys. Lett. 95 (2009) 074102.

12. J. D. Miller, M. N. Slipchenko, T. R. Meyer, H. U. Stauffer, J. R. Gord, Opt. Lett. 35 (2010) 2430-2432.

13. J. D. Miller, M. N. Slipchenko, T. R. Meyer, Opt. Express 19 (2011) 13326-13333.

14. J. D. Miller, S. Roy, M. N. Slipchenko, J. R. Gord, T. R. Meyer, Opt. Express 19 (2011) 15627-15640.

15. J. D. Miller, S. Roy, J. R. Gord, T. R. Meyer, J. Chem. Phys. 135 (2011) 201104.

16. J. D. Miller, C. E. Dedic, S. Roy, J. R. Gord, T. R. Meyer, Opt. Express 20 (2012) 50035010.

17. S. P. Kearney, D. J. Scoglietti, C. J. Kliewer, Opt. Express 21 (2013) 12327-12339.

18. S. P. Kearney, D. J. Scoglietti, Opt. Lett. 38 (2013) 833-835.

19. A. Bohlin, C. J. Kliewer, J. Chem. Phys. 138 (2013) 221101.

20. A. Bohlin, B. D. Patterson, C. J. Kliewer, J. Chem. Phys. 138 (2013) 081102.

21. B. D. Patterson, Y. Gao, T. Seeger, C. J. Kliewer, Opt. Lett. 38 (2013) 4566-4569.

22. H. U. Stauffer, J. D. Miller, S. Roy, J. R. Gord, T. R. Meyer, J. Chem. Phys. 136 (2012) 111101.

23. D. R. Richardson, D. Bangar, R. P. Lucht, Opt. Express 20 (2012) 21495-21504.

24. D. R. Richardson, R. P. Lucht, W. D. Kulatilika, S. Roy, J. R. Gord, Appl. Phys. B 104 (2011) 699-714.

25. P. Beaud, H. M. Frey, T. Lang, M. Motzkus, Chem. Phys. Lett. 344 (2001) 407-412.

26. C. N. Dennis, C. D. Slabaugh, I. G. Boxx, W. Meier, R. P. Lucht, Proc. Combust. Inst. (in press) (2014), http://dx.doi.org/10.1016/j.proci.2014.06.063

27. A. Bohlin, M. Mann, B. D. Patterson, A. Dreizler, C. J. Kliewer, Proc. Combust. Inst. (in press) (2014), http://dx.doi.org/10.1016/j.proci.2014.05.124

28. R. J. Hall, L. R. Boedeker, Appl. Opt. 23 (1984) 1340-1346.

29. P. J. Wrzesinski, H. U. Stauffer, W. D. Kulatilika, J. R. Gord, S. Roy, J. Raman Spectrosc. 44 (2013) 1344-1348.

30. W. D. Kulatilika, P. S. Hsu, H. U. Stauffer, J. R. Gord, S. Roy, Appl. Phys. Lett. 97 (2010) 081112 . 
31. C. J. Kliewer, A. Bohlin, E. Nordström, B. D. Patterson, P. E. Bengtsson, T. B. Settersten, Appl. Phys. B 108 (2012) 419-426.

32. S. Roy, P. S. Hsu, N. Jiang, J. R. Gord, W. D. Kulatilika, H. U. Stauffer, J. R. Gord, J. Chem. Phys. 138 (2013) 024201.

33. A. Bohlin, C. J. Kliewer, J. Phys. Chem. Lett. 5 (2014) 1242-1248.

34. A. Bohlin, C. J. Kliewer, Appl. Phys. Lett. 104 (2014) 031107.

35. C. E. Dedic, J. D. Miller, T. R. Meyer, Opt. Lett. 39 (2014) 6608-6611.

36. H. Tran, B. Lavorel, O. Faucher, R. Saint-Loup, P. Joubert, J. Raman Spectrosc. 34 (2003) 994-998.

37. T. Hornung, H. Skenderovic, K. L. Kompa, M. Motzkus, J. Raman Spectrosc. 35 (2004) 934-938.

38. T. Seeger, A. Leipertz, Appl. Opt. 35 (1996) 2665-2671.

39. S. Kröll, P. E. Bengtsson, D. Nilsson, Appl. Phys. B 51 (1990) 25-30.

40. A. Thumann, M. Schenk, J. Jonuscheit, T. Seeger, A. Leipertz, Appl. Opt. 36 (1997) 35003505.

41. M. Schenk, T. Seeger, A. Leipertz, Appl. Opt. 44 (2005) 5582-5593.

42. F. Vestin, P. E. Bengtsson, Proc. Combust. Inst. 32 (2009) 847-854.

43. B. D. Prince, A. Chakraborty, B. M. Prince, H. U. Stauffer, J. Chem. Phys. 125 (2006) 044502.

44. R. D. Hancock, K. E. Bertagnolli, R. P. Lucht, Combust. Flame 109 (1997) 323-331.

45. F. Xu, B. Sunderland, G. M. Faeth, Combust. Flame 108 (1997) 471-493.

46. F. Vestin, M. Afzelius, C. Brackmann, P. E. Bengtsson, Proc. Combust. Inst. 30 (2005) 1673-1680.

47. A. Bohlin. Development and application of pure rotational CARS for reactive flows, Ph.D. Thesis. Lund University, 2012.

48. G. Knopp, K. Kirch, P. Beaud, K. Mishima, H. Spitzer, P. Radi, M. Tulej, T. Gerber, J. Raman Spectrosc. 34 (2003) 989-993.

49. S. Roy, P. J. Wrzesinski, D. Pestov, M. Dantus, J. R. Gord, J. Raman Spectrosc. 41 (2009) 1194-1199.

50. A. Bohlin, C. J. Kliewer, Appl. Phys. Lett. 104 (2014) 031107.

51. P. J. Wrzesinski, D. Pestov, V. V. Lozovoy, B. Xu, S. Roy, J. R. Gord, M. Dantus, J. Raman Spectrosc. 42 (2011) 393-398.

52. A. Bohlin, C. J. Kliewer, Appl. Phys. Lett. 105 (2014) 161111.

53. S. P. Kearney, Appl. Opt. 53 (2014) 6579-6585.

54. A. C. Eckbreth, Appl. Phys. Lett. 32 (1978) 421-423.

55. I. R. Beattie, T. R. Gilson, D. A. Greenhalgh, Nature 276 (1978) 378-379.

56. F. Raoult, A. C. I. Boscheron, D. Husson, C. Sauteret, A. Modena, V. Malka, F. Dorchies, A. Migus, Opt. Lett. 23 (1998) 1117-1119.

57. O. E. Martinez, IEEE J. Quantum Electron. 23 (1987) 59-64.

58. A. M. Weiner, Optics Communications 284 (2011) 3669-3692.

59. H. U. Stauffer, J. D. Miller, M. N. Slipchenko, T. R. Meyer, B. D. Prince, S. Roy, J. R. Gord, J. Chem. Phys. 140 (2014) 024316.

60. R. E. Palmer, SAND89-8206, Sandia National Laboratories: Livermore, CA (1989).

61. M. C. Drake, Opt. Lett. 7 (1982) 440-441.

62. A. Bohlin, P. E. Bengtsson, M. Marrocco, J. Raman Spectrosc. 42 (2011) 1843-1847. 
63. L. Martinsson, P. E. Bengtsson, M. Aldén, S. Kröll, J. Bonamy, J. Chem. Phys. 99 (1993) 2466-2477.

64. M. Schenk, A. Thumann, T. Seeger, A. Leipertz, Appl. Opt. 37 (1998) 5659-5671.

65. http://liiscience.org/target flames

66. http://www.adelaide.edu.au/cet/isfworkshop/

67. A. Bohlin, F. Vestin, J. Bonamy, P. Joubert, P. E. Bengtsson, J. Raman Spectrosc. 41 (2010) 875-881.

68. S. P. Kearney, M. N. Jackson, AIAA J. 45 (2007) 2947-2956.

69. M. Aldén, P. E. Bengtsson, H. Edner, Appl. Opt. 25 (1986) 4493-4500. 


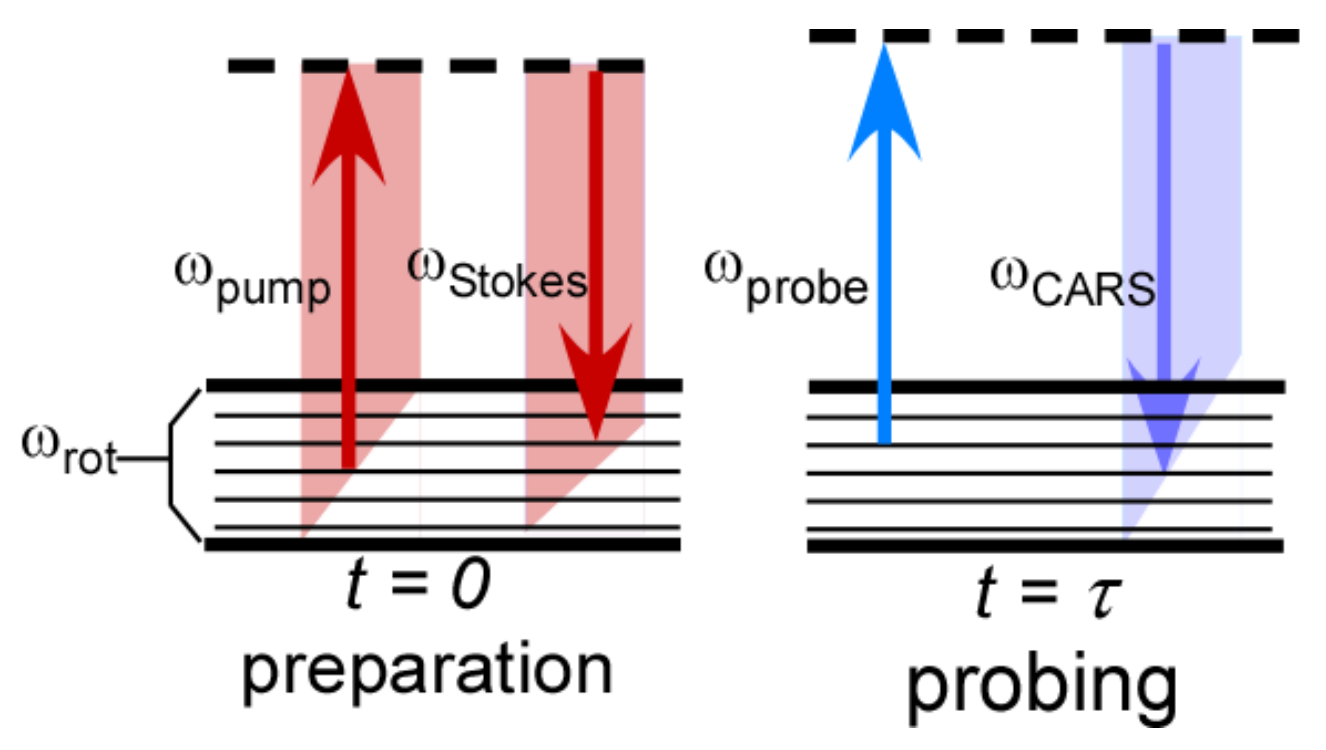




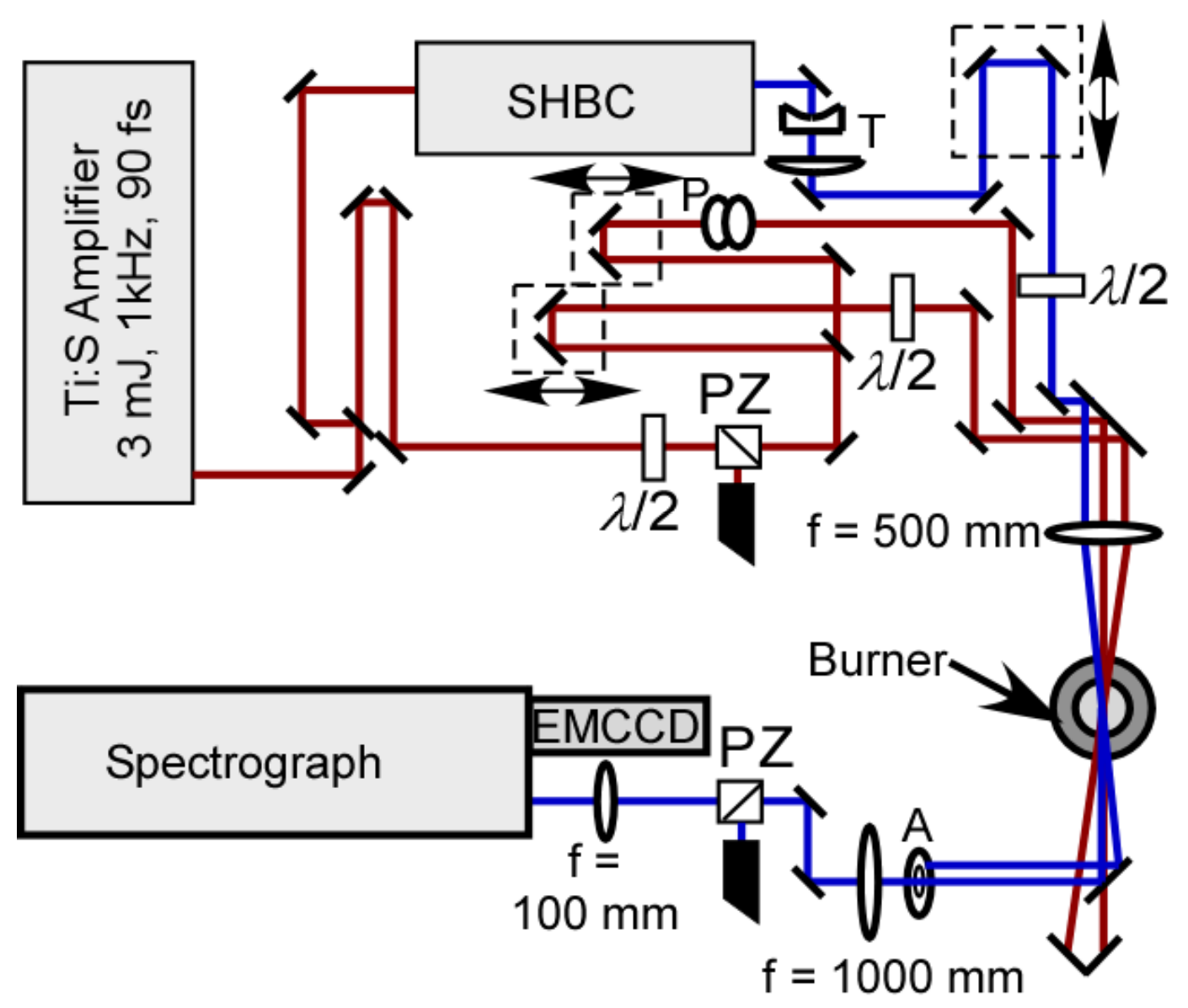



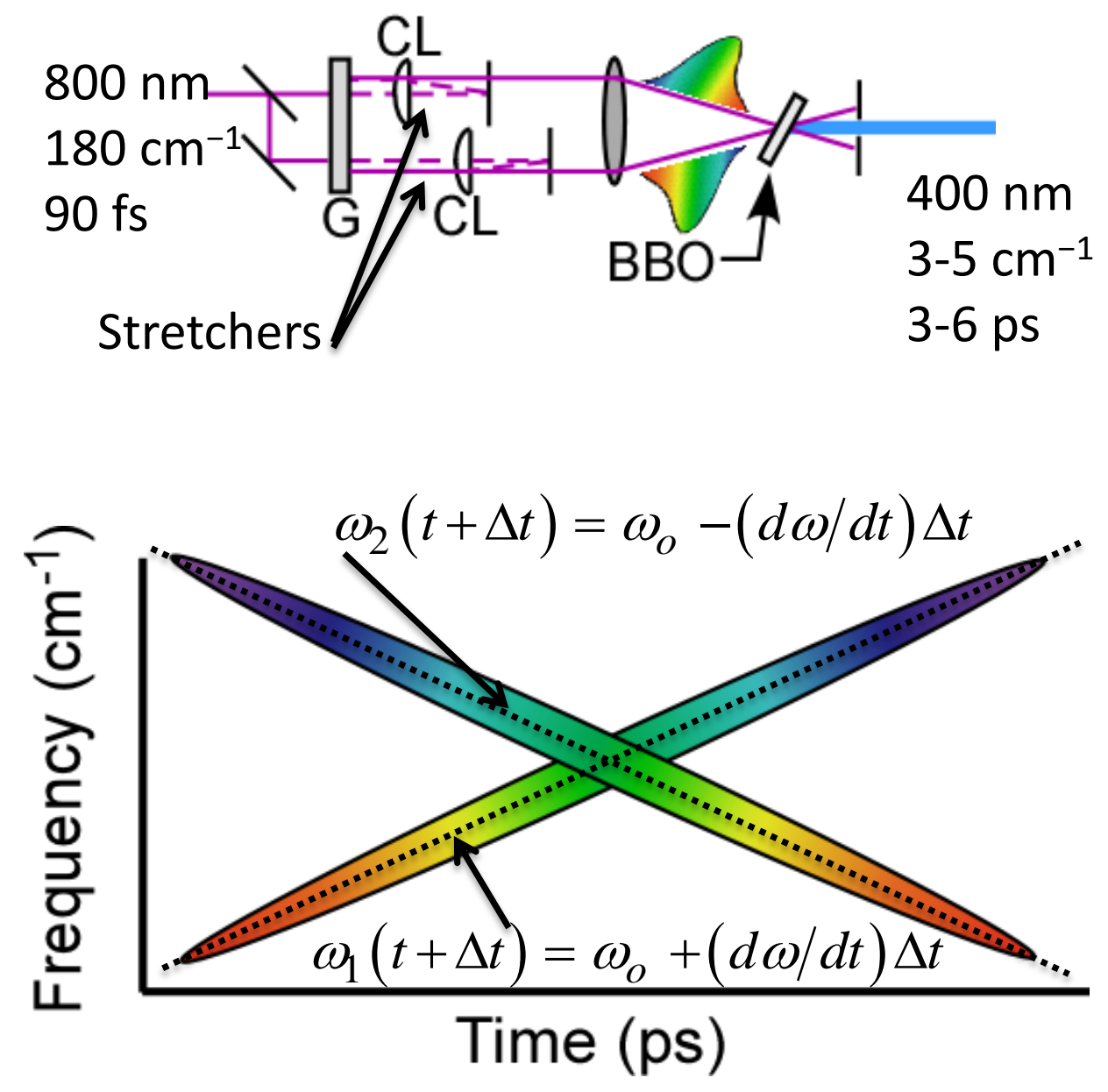


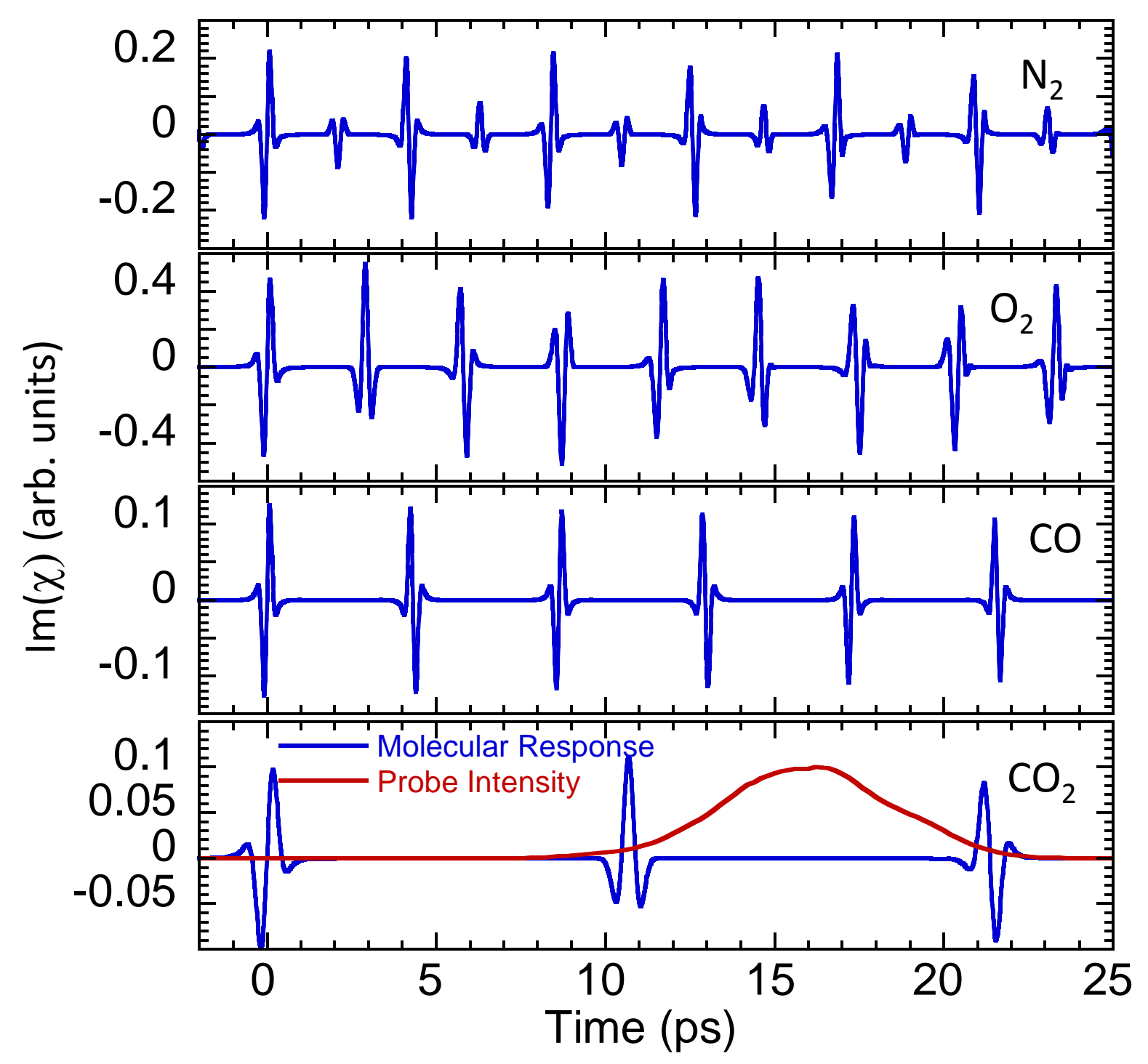




\section{Figure 5}

Click here to download Figure: Figure5.pptx

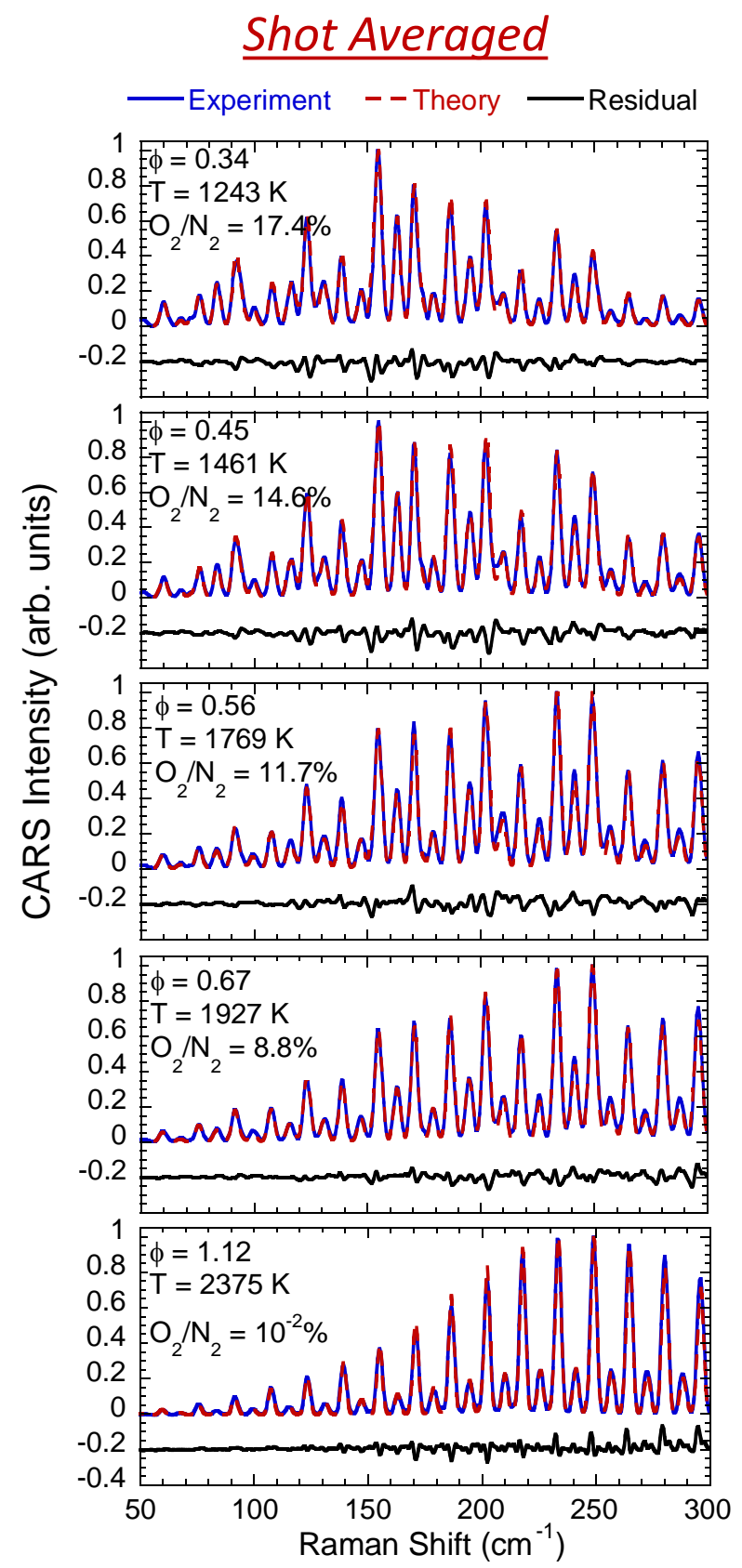

Single-Shot at $1 \mathrm{kHz}$

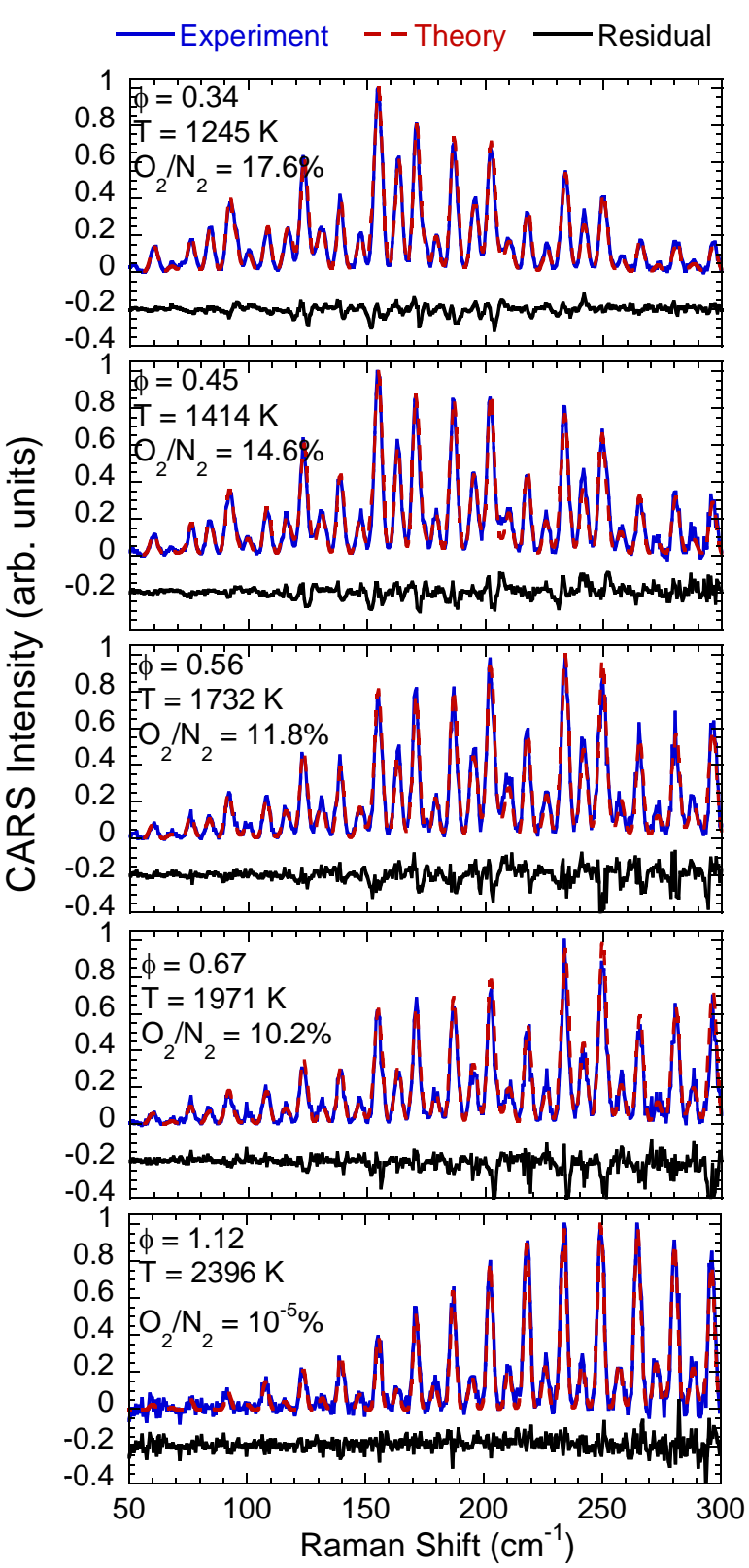




\section{igure 6}

Click here to download Figure: Figure6.pptx
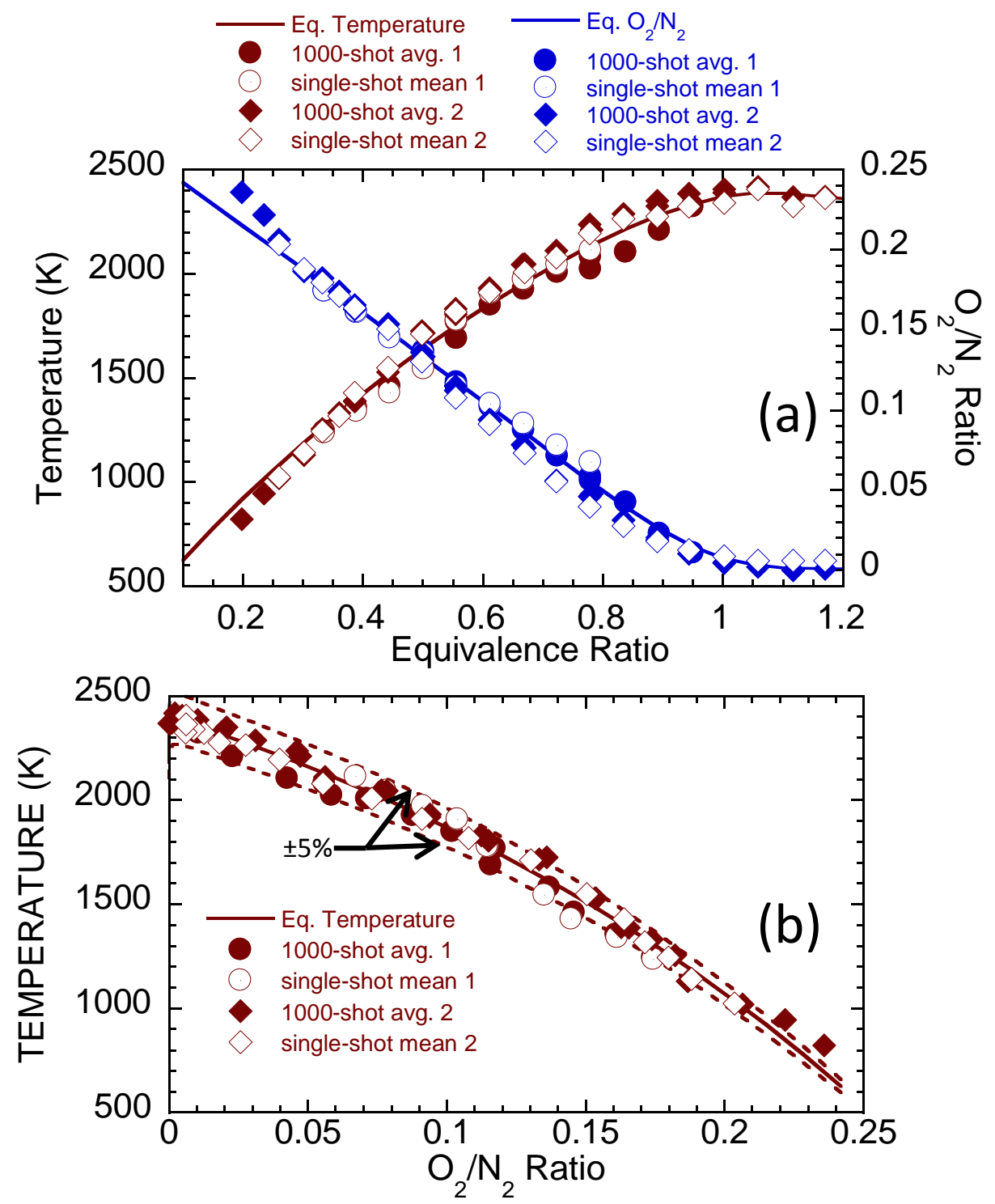

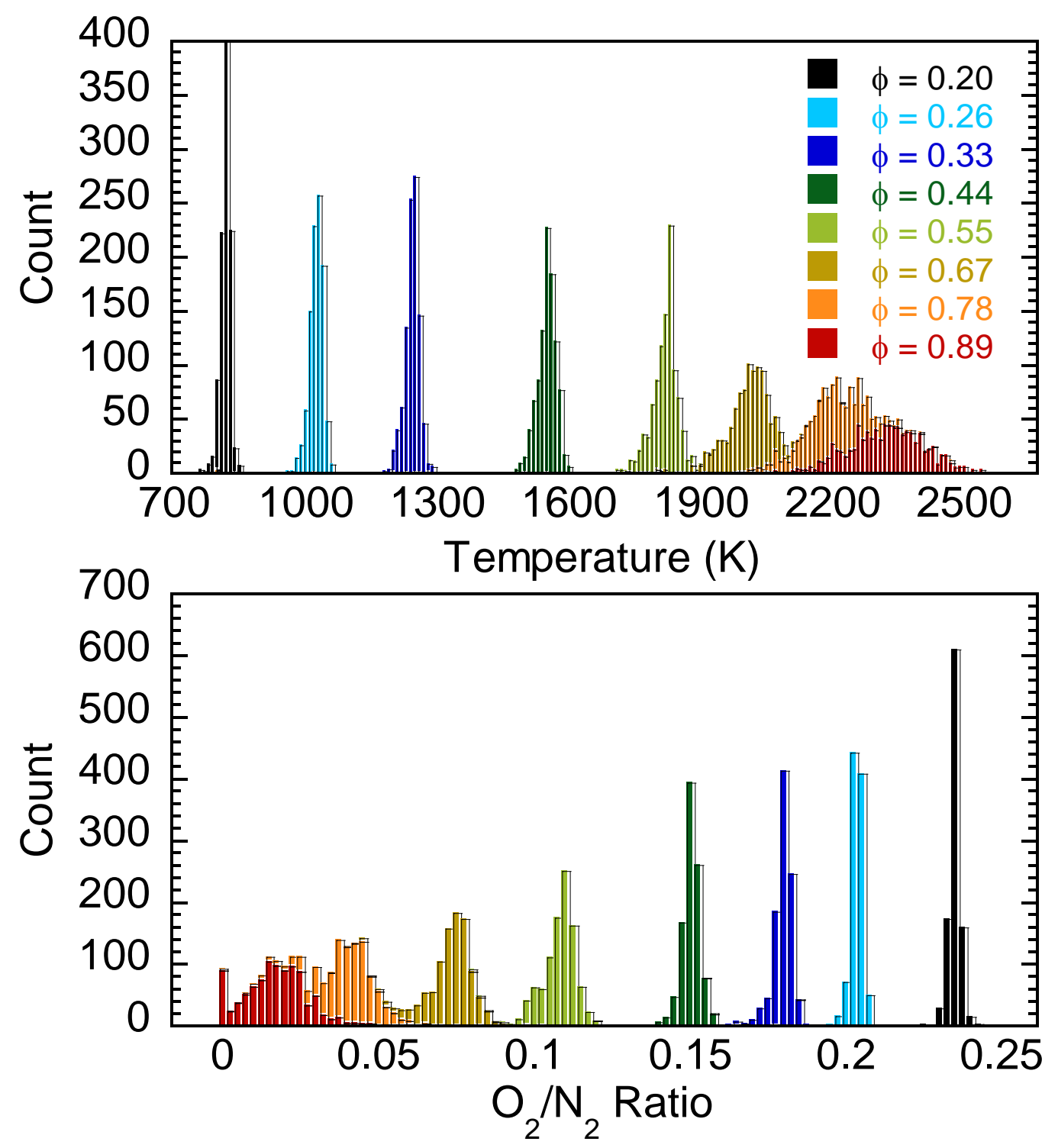


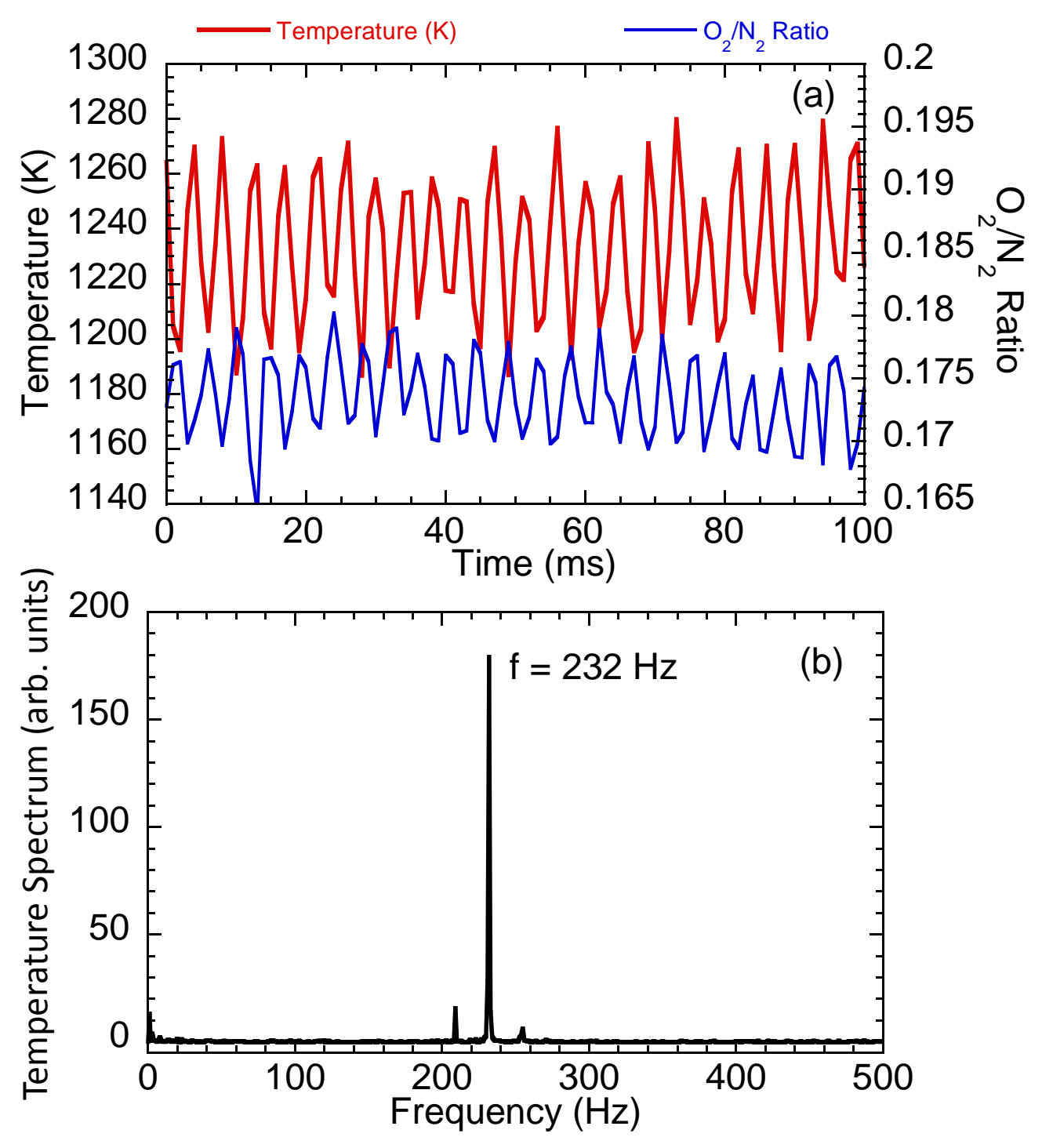




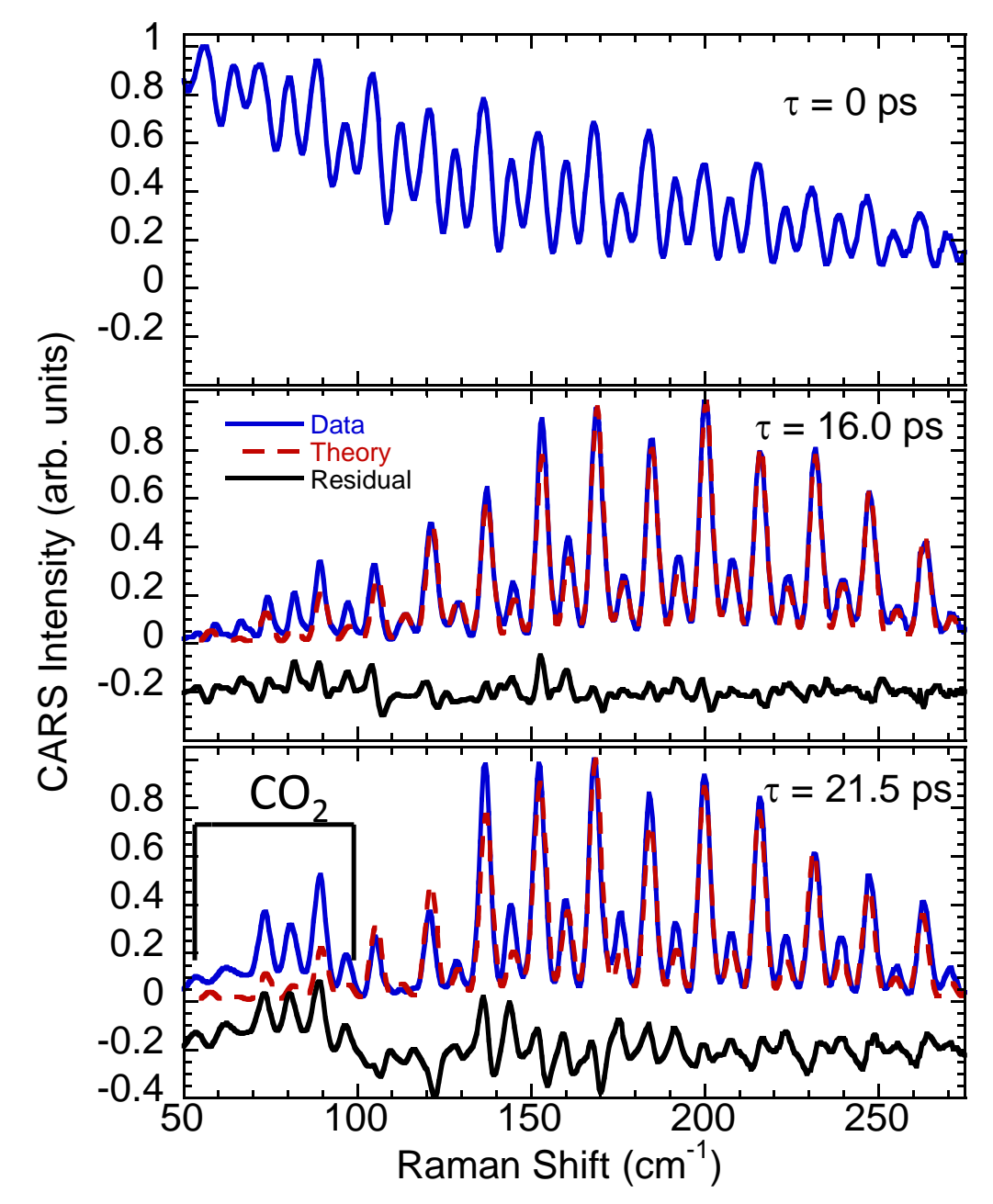




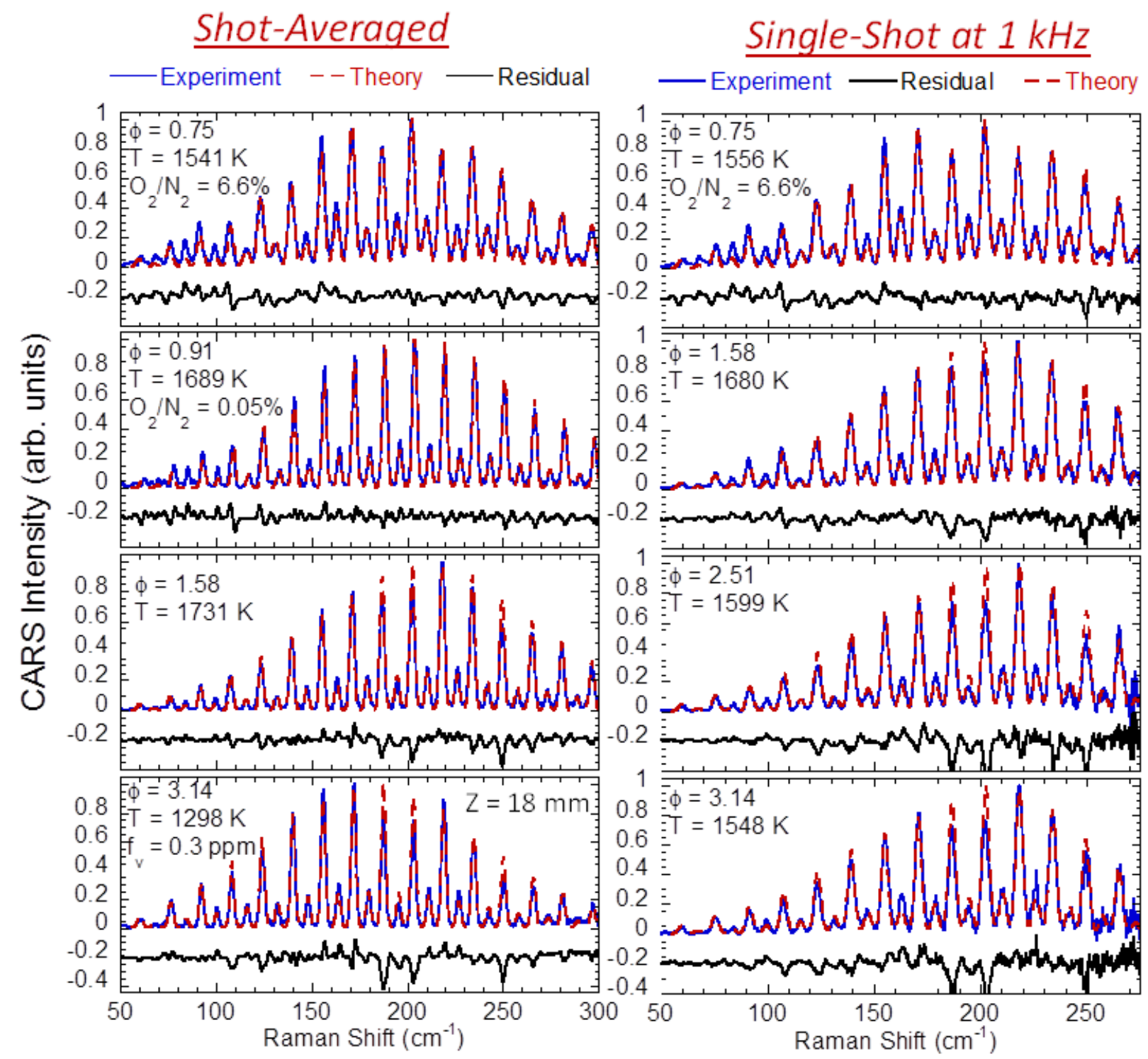



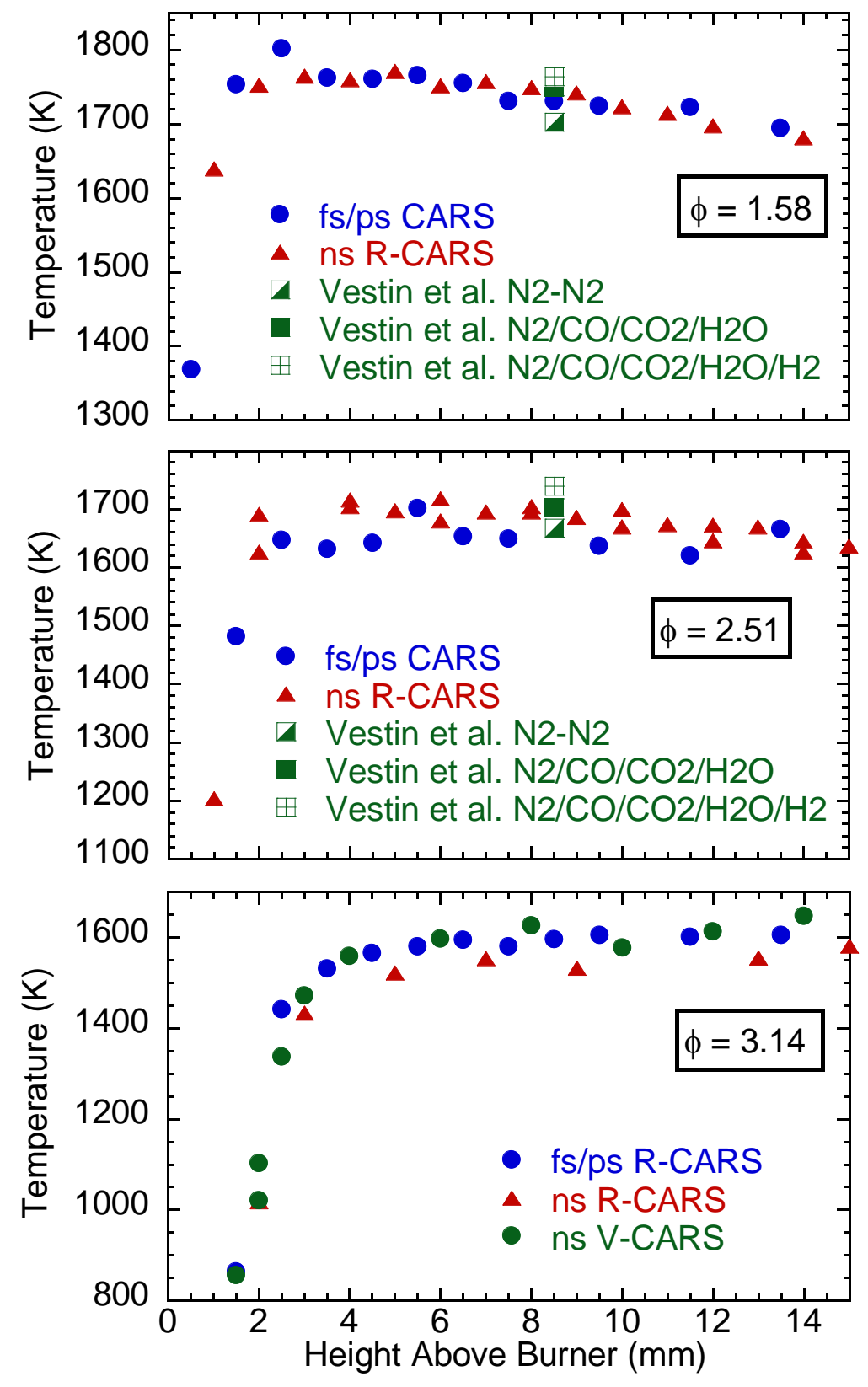


\section{Figure 12}

Click here to download Figure: Figure12.pptx

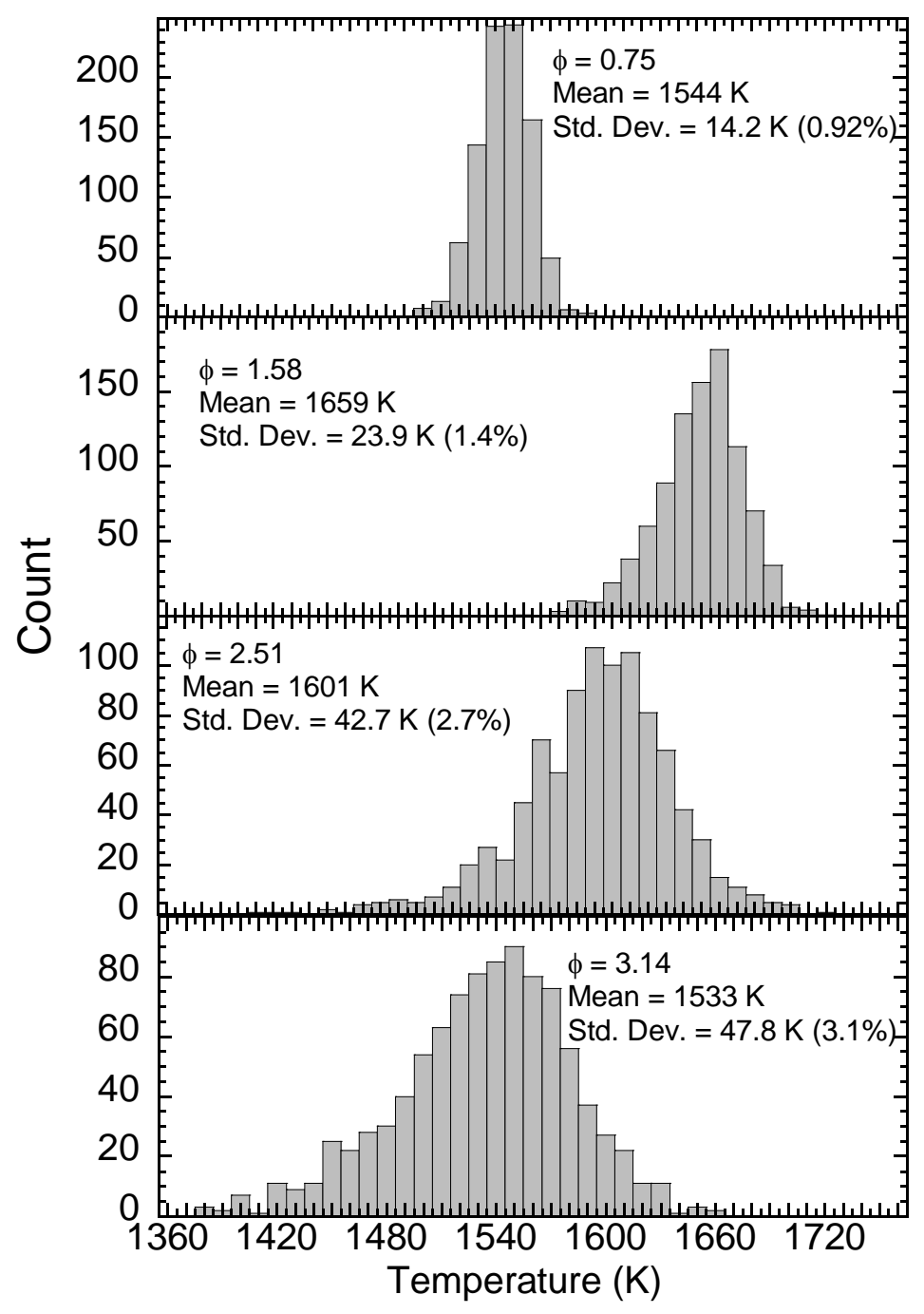



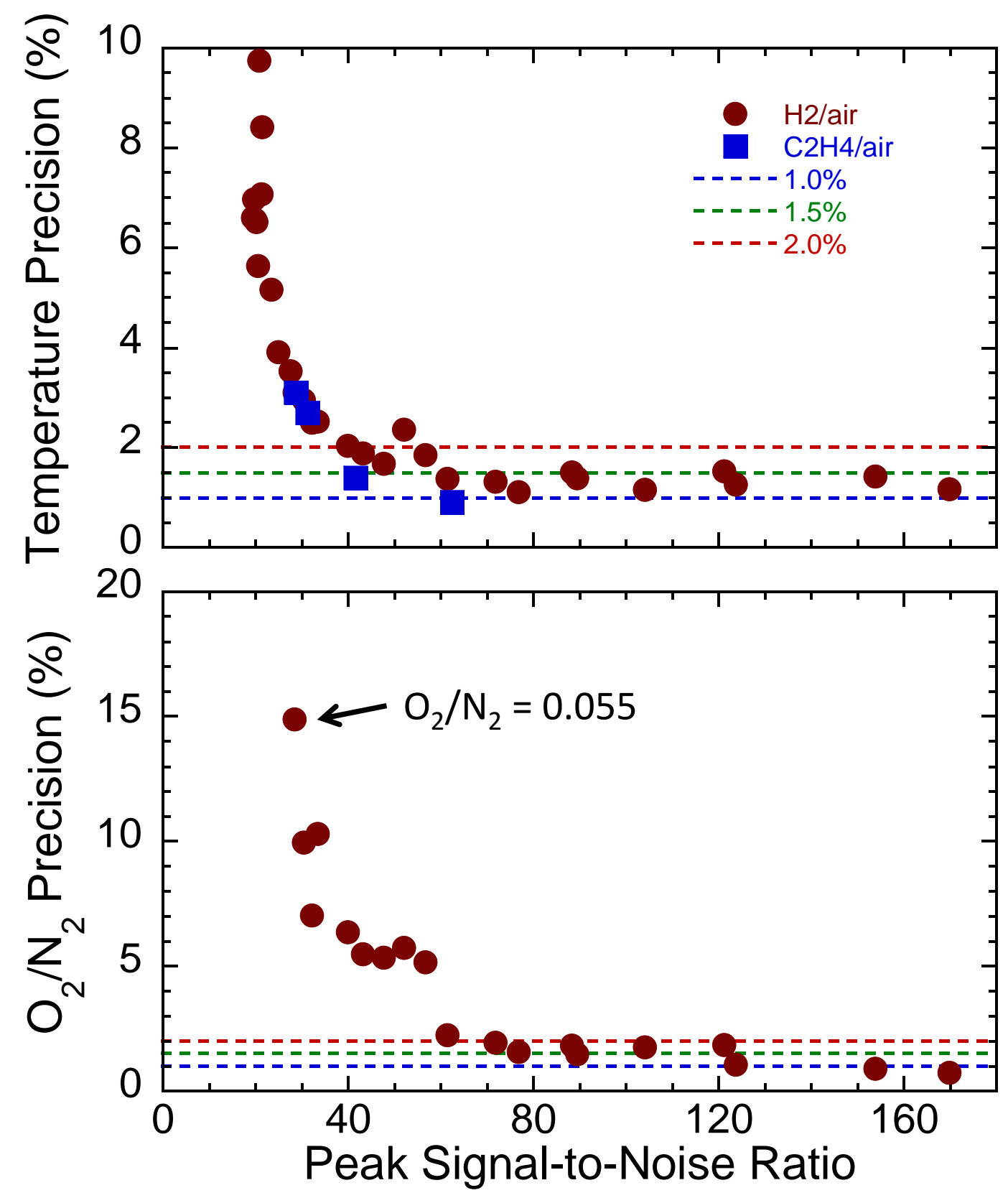